\title{
SEMI-CLASSICAL ANALYSIS OF A CONJOINT CROSSING OF THREE SYMMETRIC MODES*
}

\author{
CLOTILDE FERMANIAN KAMMERER ${ }^{\dagger}$ AND VIDIAN ROUSSE ${ }^{\ddagger}$
}

\begin{abstract}
In this article we focus on a semiclassical Schrödinger equation with matrix-valued potential presenting a symmetric conjoint crossing of three eigenvalues. The potential we consider is well-known in the chemical literature as a pseudo Jahn-Teller potential. We analyze the energy transfers which occur between the three modes in terms of Wigner measures.
\end{abstract}

Key words. Semi-classical analysis, Born-Oppenheimer approximation, Schrödinger equation, Wigner measures, eigenvalue crossings.

AMS subject classifications. 35Q40 (81Q20, 35B27)

1. Introduction. We consider the following Schrödinger equation

$$
\left\{\begin{array}{l}
i \varepsilon \partial_{t} \psi^{\varepsilon}(q, t)=\left(-\frac{\varepsilon^{2}}{2} \Delta_{q}+V(q)\right) \psi^{\varepsilon}(q, t), \quad(q, t) \in \mathbf{R}^{2} \times \mathbf{R} \\
\psi^{\varepsilon}(q, 0)=\psi_{0}^{\varepsilon}(q) \in L^{2}\left(\mathbf{R}^{2}, \mathbf{C}^{3}\right),
\end{array}\right.
$$

where $\varepsilon>0$ is the semi-classical parameter and where the matrix-valued potential $V(q)=V_{P J T}(q)$ is

$$
\forall q \in \mathbf{R}^{2}, \quad V_{P J T}(q)=\left(\begin{array}{ccc}
q_{1} & 0 & q_{2} / \sqrt{2} \\
0 & -q_{1} & q_{2} / \sqrt{2} \\
q_{2} / \sqrt{2} & q_{2} / \sqrt{2} & 0
\end{array}\right) .
$$

Therefore, the potential $V$ is subquadratic and the operator $-\frac{\varepsilon^{2}}{2} \Delta_{q}+V(q)$ is essentially self-adjoint on the Schwartz space. Such systems naturally arise in quantum chemistry when studying the dynamics of large molecules in the frame of BornOppenheimer approximation. The parameter $\varepsilon$ is small because it takes into account the ratio of the mass of one electron and of the averaged mass of the nuclei (see [1] or [19] for the derivation of such equations). The solution $\psi^{\varepsilon}(t)$ does not have any direct physical interpretation but only quadratic functions of it: for example, the quantity $\left|\psi^{\varepsilon}(q, t)\right|^{2}$ - which is the norm in $\mathbf{C}^{3}$ of the vector $\psi^{\varepsilon}(q, t)$ - is called the position density and gives the probability of finding the nuclei in the configuration $q \in \mathbf{R}^{2}$ at time $t$. One is interested in the asymptotic description for the time evolution of the matrix-valued Wigner function of $\psi^{\varepsilon}(q, t)$,

$$
W^{\varepsilon}\left(\psi^{\varepsilon}(t)\right)(q, p)=(2 \pi)^{-d} \int_{\mathbf{R}^{2}} \psi^{\varepsilon}\left(q-\frac{\varepsilon}{2} v, t\right) \otimes \bar{\psi}^{\varepsilon}\left(q+\frac{\varepsilon}{2} v, t\right) \mathrm{e}^{i v \cdot p} \mathrm{~d} v
$$

which plays the role of a generalized probability density on phase space. Indeed, the action of the Wigner function against compactly supported smooth test functions $a \in \mathcal{C}_{c}^{\infty}\left(\mathbf{R}^{4}, \mathbf{C}^{3 \times 3}\right)$ is simply expressed in terms of the semi-classical pseudodifferential

\footnotetext{
${ }^{*}$ Received February 28, 2010; accepted for publication June 18, 2010.

${ }^{\dagger}$ Université Paris Est, UFR des Sciences et Technologie, 61, avenue du Général de Gaulle, 94010 Créteil Cedex, France (clotilde.fermanian@univ-paris12.fr).

$\ddagger$ Université Paris Est, UFR des Sciences et Technologie, 61, avenue du Général de Gaulle, 94010 Créteil Cedex, France (vidian.rousse@univ-paris12.fr).
} 
operator with symbol $a$, which is defined for $\psi \in L^{2}\left(\mathbf{R}^{2}, \mathbf{C}^{3}\right)$ by

$$
\mathrm{op}_{\varepsilon}(a) \psi(q)=(2 \pi \varepsilon)^{-d} \int_{\mathbf{R}^{4}} a\left(\frac{q+v}{2}, p\right) \mathrm{e}^{\frac{i}{\varepsilon} p \cdot(q-v)} \psi(v) \mathrm{d} v \mathrm{~d} p .
$$

We then have $\operatorname{tr} \int_{\mathbf{R}^{4}} W^{\varepsilon}(\psi)(q, p) a(q, p) \mathrm{d} q \mathrm{~d} p=\left(\operatorname{op}_{\varepsilon}(a) \psi, \psi\right)_{L^{2}\left(\mathbf{R}^{2}, \mathbf{C}^{3}\right)}$. For example, one recovers the position density by $|\psi(q)|^{2}=\operatorname{tr} \int_{\mathbf{R}^{2}} W^{\varepsilon}(\psi)(q, p) \mathrm{d} p$. We are concerned here with the description of the weak limits of the Wigner transform, these distributions are matrix-valued measures called Wigner measures or semi-classical measures (see [25], [17] or [18]). Besides, these matrix-valued measures $\mu=\left(\mu_{i, j}\right)$ are positive in the sense that their diagonal elements $\mu_{i, i}$ are positive Radon measures and their off-diagonal elements $\mu_{i, j}$ for $i \neq j$ are absolutely continuous with respect to $\mu_{i, i}$ and $\mu_{j, j}$.

The potential $V_{P J T}(q)$ arises in models of quantum chemistry: it is the simplest pseudo Jahn-Teller Hamiltonian as introduced in [8] Chapter 10 (see also [30] or [10] for example). Its main feature is that its eigenvalues are $\sqrt{q_{1}^{2}+q_{2}^{2}},-\sqrt{q_{1}^{2}+q_{2}^{2}}$ and 0 . They are symmetric: the eigenvalue 0 is exactly the half sum of the two other eigenvalues. Besides, they are of multiplicity 1 as long as $\left(q_{1}, q_{2}\right) \neq(0,0)$ and they simultaneously cross on the point $\left\{q_{1}=q_{2}=0\right\}$. We will say that $V$ presents a conjoint symmetric crossing in $q=(0,0)=0$. The appellation of pseudo Jahn-Teller potential is a reference to what is called Jahn-Teller potential in quantum chemistry, namely the 2 by 2 potential of the form $\left(\begin{array}{cc}q_{1} & q_{2} \\ q_{2} & -q_{1}\end{array}\right)$ to which numerous mathematical works have been devoted. Then, another simple realization of a symmetric conjoint crossing is the matrix

$$
V_{J T}\left(q_{1}, q_{2}\right)=\left(\begin{array}{ccc}
q_{1} & q_{2} & 0 \\
q_{2} & -q_{1} & 0 \\
0 & 0 & 0
\end{array}\right) .
$$

Both matrices $V_{J T}$ and $V_{P J T}$ (defined in (2)) have the same symmetric eigenvalues and the behavior of solutions to (1) with the Jahn-Teller potential $V_{J T}$ is well understood. Indeed, in that situation, the third mode evolves at leading order independently from the two other ones which interact following the well-known process of conical intersections (see [22], [31], [19], [20], [14] for example). However, the situation is different for the pseudo Jahn-Teller potential $V_{P J T}(q)$, we will see that the three modes will interact altogether; note also that for this potential, the three spectral projectors are singular on $\left\{q_{1}=q_{2}=0\right\}$ while $V_{J T}(q)$ has a smooth eigenprojector (for the mode 0 ). While an important literature has been devoted to two by two crossings, there are very few results on conjoint crossing of three eigenvalues. The aim of this work is to analyze on a simple model the mechanism of a symmetric crossing of three eigenvalues.

We shall describe how the three modes interact together for the pseudo Jahn-Teller potential $V_{P J T}(q)$ by studying the branching of Wigner measures on the crossing set $\left\{q_{1}=q_{2}=0\right\}$. We explain our result in the following section. The main ingredients of the proof are a normal form result given in Section 3 and a scattering theorem in Section 4. The normal form reduces the analysis of the crossing to the study of a 
system very close to the system of three ordinary differential equations

$$
i \varepsilon \partial_{s} u+\left(\begin{array}{ccc}
s & 0 & z / \sqrt{2} \\
0 & -s & z / \sqrt{2} \\
z / \sqrt{2} & z / \sqrt{2} & 0
\end{array}\right)=0
$$

The behavior of solutions of this system as $\varepsilon$ goes to 0 is analyzed in Section 4 . This system has been already studied by physicists ([2] and [3]); with their arguments, one can find the same result than ours, however, we give here a different and slightly shorter proof (see Remark 7). These two results allow to understand the evolution of Wigner measures of families of solutions to (1) in Section 5.

This paper is the first step in the understanding of symmetric conjoint crossings. For applications in quantum chemistry, it would be interesting to be able to treat general potential $V(q)$ presenting a conjoint crossing of symmetric eigenvalues on a codimension 2 submanifold. Then, it is likely that the potentials $V_{J T}$ and $V_{P J T}$ will play a crucial role as toys model. This motivates the work performed in this paper. However, for the moment, we are not able to treat potentials more generals than $V_{P J T}$ even in simple situations. For example, if one simply adds a scalar potential to $V_{P J T}$ by chosing $V(q)=V_{P J T}(q)+v(q) \mathrm{Id}$, the second step of the normal form (see the gauge transform, Section 3.1 below) generates perturbative terms that we are not able to treat for the moment.

Finally, let us mention that the application to quantum chemistry we have in mind concerns the elaboration of algorithms modelizing the evolution of the Wigner transform of families of solutions of Schrödinger equation (1). These algorithms have been extensively developed since the 70's and the pioneer work of Tully and Preston (see [29]). The algorithms of [24] and [15] which are the first one presenting a mathematical proof rely on normal forms results of [4], [5] and [12] and on the precise analysis of the propagation of Wigner measures through the crossing performed in [14] and [11]. Therefore, the result of this article concerning the branching of Wigner measures has important consequences for numerics in quantum chemistry: one can construct an algorithm for the potential $V_{P J T}(q)$ exactly as in [24] and its convergence is guaranteed by the Theorem 1 in the same manner than the algorithm of [24] relies on the branching theorem of [13]. The diagrams illustrating the statement of the main result in the next section are obtained that way.

2. Main result. Let us begin with some notations. For $\ell \in\{0,+1,-1\}$, we denote by $\Pi^{\ell}(q)$ the eigenprojector associated with $\ell|q|$ and we set

$$
\lambda^{\ell}(q, p, \tau)=\tau+\frac{|p|^{2}}{2}+\ell|q|
$$

The indices $\ell \in\{0,+1,-1\}$ will be sometimes shortened into \pm whenever $\ell= \pm 1$. We consider the Hamiltonian vector fields

$$
H_{\lambda^{\ell}}=\partial_{\tau}+p \cdot \nabla_{q}-\ell \frac{q}{|q|} \cdot \nabla_{p}, \quad \ell \in\{0,+1,-1\}
$$

and their integral curves $\rho_{s}^{\ell}$ which are called classical trajectories. They are of the form

$$
\rho_{s}^{\ell}=\left(q_{s}^{\ell}, s+t_{0}, p_{s}^{\ell}, \tau_{0}\right) \text { with } \dot{q}_{s}^{\ell}=p_{s}^{\ell}, \quad \dot{p}_{s}^{\ell}=\ell \frac{q_{s}^{\ell}}{\left|q_{s}^{\ell}\right|} \text { and } \rho_{0}^{\ell}=\left(q_{0}, t_{0}, p_{0}, \tau_{0}\right) .
$$


Because of the singularity in $q=0$, something has to be said for the \pm mode: it is proved in [13] that under the assumption

$$
p_{0} \neq 0
$$

there exists a unique curve $\left(q_{s}^{ \pm}, p_{s}^{ \pm}\right)$solution of the system of ordinary equations above and such that $q_{0}^{ \pm}=q_{0}$ and $p_{0}^{ \pm}=p_{0}$. Moreover, if $q_{0}=0$, the + trajectory (resp. - trajectory) issued from $\left(q_{0}, p_{0}\right)$ smoothly continues the - trajectory (resp. + trajectory) arriving at $\left(q_{0}, p_{0}\right)$. Finally, we emphasize a specific feature of these trajectories: there exists an hypersurface containing all the curves which pass through the crossing set, namely the singular hypersurface

$$
I=\{q \wedge p=0\}
$$

where for two vectors $z=\left(z_{1}, z_{2}\right)$ and $z^{\prime}=\left(z_{1}^{\prime}, z_{2}^{\prime}\right)$ of $\mathbf{R}^{2}, z \wedge z^{\prime}$ denotes their wedge product:

$$
z \wedge z^{\prime}=z_{1} z_{2}^{\prime}-z_{2} z_{1}^{\prime}
$$

Observe that $I$ is a smooth hypersurface close to points $\rho_{0}=\left(q_{0}, t_{0}, p_{0}, \tau_{0}\right)$ such that $p_{0} \neq 0$. Note also that this assumption implies that the classical trajectories are transverse to the crossing set. It is under this assumption that most results on crossings are obtained (for example in [19], [20], [13] or [14]; for a result in case of tangency see [9]).

We work in space-time variables and call crossing set the points of the phase space where there is an eigenvalue crossing namely the set

$$
S=\left\{\tau+\frac{|p|^{2}}{2}=0, \quad q=0\right\}=\{q=0\} \cap \Sigma,
$$

where $\Sigma$ denotes the characteristic set:

$$
\Sigma=\Sigma^{+} \cup \Sigma^{-} \cup \Sigma^{0} \text { with } \Sigma^{\ell}=\left\{\lambda^{\ell}(q, p, \tau)=0\right\}
$$

being the energy surface for the mode $\ell$. The Wigner measures of families $\left(\psi^{\varepsilon}\right)$ are supported on the energy surfaces. More precisely, it is proved in [18] that outside the crossing set, one has

$$
\mu=\mu^{+} \Pi^{+}+\mu^{-} \Pi^{-}+\mu^{0} \Pi^{0}
$$

where for $\ell \in\{0,+1,-1\}$, the measures $\mu^{\ell}$ are scalar positive Radon measure such that for all $a \in \mathcal{C}_{0}^{\infty}\left(\mathbf{R}^{6}, \mathbf{C}\right)$,

$$
\left(\operatorname{op}_{\varepsilon}\left(a \Pi^{\ell}\right) \psi^{\varepsilon}, \psi^{\varepsilon}\right)_{L^{2}\left(\mathbf{R}^{3}, \mathbf{C}^{3}\right)} \underset{\varepsilon \rightarrow 0}{\longrightarrow} \int_{\mathbf{R}^{6}} a(q, t, p, \tau) \mathrm{d} \mu^{\ell}(q, t, p, \tau) .
$$

Besides, the measures $\mu^{\ell}$ are supported on $\Sigma^{\ell}$ and invariant through the Hamiltonian flow associated with $H_{\lambda^{\ell}}$ for all $\ell \in\{0,-1,+1\}$.

It is well known that the transitions between two modes cannot be described only in terms of Wigner measures (see [13]). The phenomenon of the transitions is more intricate and requires a second level of observation. Indeed, the transitions are determined by the way the families $\left(\psi^{\varepsilon}(t)\right)$ concentrate on the trajectories arriving at the crossing with respect to the scale $\sqrt{\varepsilon}$. For this reason, one uses two-scale Wigner measures 
associated with the hypersurface $I$ because all the trajectories passing through $S$ are contained in $I$.

Following [26] and [13], we consider observables $a=a(q, t, p, \tau, \eta) \in \mathcal{C}^{\infty}\left(\mathbf{R}^{7}, \mathbf{C}^{3,3}\right)$ which are compactly supported outside $\{p=0\}$ in the variables $(q, t, p, \tau)$, uniformly with respect to the variable $\eta$ and coincide for $|\eta|>R_{0}$ for some $R_{0}>0$ with a function homogeneous of degree 0 in the variable $\eta$. We will denote by $\mathcal{A}$ the vector space of such functions. Then, once given the hypersurface $I$, one associates with $a$ the pseudodifferential operator

$$
\mathrm{op}_{\varepsilon}{ }^{I}(a)=\mathrm{op}_{\varepsilon}\left(a\left(q, t, p, \tau, \frac{q \wedge p}{\sqrt{\varepsilon}}\right)\right) .
$$

Consider the scaling operator $T_{\varepsilon}$ defined by

$$
\forall f \in L^{2}\left(\mathbf{R}^{2}\right), \quad T_{\varepsilon} f(q)=\sqrt{\varepsilon} f(q \sqrt{\varepsilon}),
$$

then

$$
T_{\varepsilon} \mathrm{op}_{\varepsilon}{ }^{I}(a) T_{\varepsilon}^{*}=\mathrm{op}_{1}(a(q \sqrt{\varepsilon}, t, p \sqrt{\varepsilon}, \tau \varepsilon, \sqrt{\varepsilon} q \wedge p))
$$

and Calderón-Vaillancourt's Theorem shows that the family of operators op $_{\varepsilon}{ }^{I}(a)$ is uniformly bounded with respect to $\varepsilon$ on $L_{l o c}^{2}\left(\mathbf{R}^{3}\right)$. A two-scale Wigner measure associated with the concentration of the family $\left(\psi^{\varepsilon}\right)$ on $I$ is a positive Radon measure on the compactified normal bundle to $I$ such that

$$
\begin{aligned}
& \left(\operatorname{op}_{\varepsilon}{ }^{I}(a) \psi^{\varepsilon}, \psi^{\varepsilon}\right)_{L^{2}\left(\mathbf{R}^{3}, \mathbf{C}^{3}\right)} \underset{\varepsilon \rightarrow 0}{\longrightarrow} \\
& \quad \int_{\bar{N}(I)} a(q, t, p, \tau, \eta) \mathrm{d} \nu(q, t, p, \tau, \eta)+\int_{I^{c}} a\left(q, t, p, \tau, \frac{q \wedge p}{|q \wedge p|} \infty\right) \mathrm{d} \mu(q, t, p, \tau) .
\end{aligned}
$$

We recall that the fibre of the normal bundle to $I$ is obtained above a point $\rho$ of $I$ by taking the quotient the vector space $T_{\rho}\left(T^{*} \mathbf{R}^{3}\right)$ by the tangent to $I, T_{\rho} I$. The fibre above $I$ is a dimension 1 vector space $N_{\rho}(I)$ and one gets the fibre of the compactified bundle to $I$ by adding two points at infinity; then, this fibre is isomorphic to $\overline{\mathbf{R}}$. The function $a$ being homogeneous in the variable $\eta$, it defines a function on $\bar{N}_{\rho}(I)$. We point out that $\mu$ above $I$ is the projection of $\nu$ on $I$ :

$$
\mu(q, t, p, \tau) \mathbf{1}_{I}=\int_{\overline{\mathbf{R}}} \nu(q, t, p, \tau, \mathrm{d} \eta) .
$$

Since $\mu$ is determined outside $I$ by the Wigner measure of the data (because of the invariance of $\mu^{\ell}$ through $H_{\lambda^{\ell}}$ outside $S$ ), we focus on what happens above $I$ and thus on the measure $\nu$.

Similarly to Wigner measures, the two-scale Wigner measures satisfy localization and propagation properties: it is proved in [13] that outside $S$,

$$
\nu=\nu^{0} \Pi^{0}+\nu^{+} \Pi^{+}+\nu^{-} \Pi^{-}
$$

where for any $\ell \in\{0,+1,-1\}$, the scalar positive Radon measures $\nu^{\ell}$ are supported on $\Sigma^{\ell}$ and propagate along the linearized Hamiltonian flow induced on $\bar{N}(I)$ by the Hamiltonian vector fields $H_{\lambda^{\ell}}$. We are now able to describe the branching of two scale Wigner measures close to a point $\rho_{0}=\left(q_{0}, t_{0}, p_{0}, \tau_{0}\right)$ such that $p_{0} \neq 0$. Indeed, since $p_{0} \neq 0$, the classical trajectories are transverse to the crossing set $S$ and for any 
$\ell \in\{0,+1,-1\}$, the measures $\nu^{\ell}$ have traces on $S$. Let us denote by $\nu_{\text {in }}^{\ell}$ (resp. $\nu_{\text {out }}^{\ell}$ ) the traces of $\nu^{\ell}$ on the in-going (resp. out-going) side of $S$. For expressing the link between the traces, we need to choose coordinates on $\bar{N}(I)$ : for $\rho=(q, t, p, \tau) \in I$, we characterize the class in $N_{\rho}(I)$ of the vector $\delta \rho=(\delta q, \delta t, \delta p, \delta \tau)$ of $T_{\rho} I$ by the coordinate

$$
\eta=\delta q \wedge p
$$

One is able to link these traces if they are mutually singular in the sense that one has $\nu_{i n}^{\ell} \perp \nu_{i n}^{\ell^{\prime}}$ for any $\ell, \ell^{\prime} \in\{0,+1,-1\}, \ell \neq \ell^{\prime}$.

TheOrem 1. Above $\rho_{0}=\left(q_{0}, t_{0}, p_{0}, \tau_{0}\right) \in S$ with $p_{0} \neq 0$ and under the condition that $\nu_{\text {in }}^{0}, \nu_{\text {in }}^{+}$and $\nu_{\text {in }}^{-}$are mutually singular on $\{|\eta|<+\infty\}$, one has

$$
\left(\begin{array}{c}
\nu_{\text {out }}^{+} \\
\nu_{\text {out }}^{-} \\
\nu_{\text {out }}^{0}
\end{array}\right)=\left(\begin{array}{ccc}
(1-T)^{2} & T^{2} & 2 T(1-T) \\
T^{2} & (1-T)^{2} & 2 T(1-T) \\
2 T(1-T) & 2 T(1-T) & (1-2 T)^{2}
\end{array}\right)\left(\begin{array}{c}
\nu_{\text {in }}^{+} \\
\nu_{\text {in }}^{-} \\
\nu_{\text {in }}^{0}
\end{array}\right)
$$

where

$$
T(p, \eta)=\exp \left(-\frac{\pi \eta^{2}}{2|p|^{3}}\right)
$$

The coefficient $T(p, \eta)$ is very close to the Landau-Zener coefficient $\exp \left(-\frac{\pi \eta^{2}}{|p|^{3}}\right)$ which appears for crossings of two modes (see [22], [31], [20] and [13]).

We could also have expressed the transitions through the crossing set by considering the two-scale Wigner measures associated with the concentration of $\left(\psi^{\varepsilon}\right)$ on the sets $J_{\text {in }}^{\ell}\left(\right.$ resp. $J_{\text {out }}^{\ell}$ ) consisting of all the trajectories for the mode $\ell$ arriving to (resp. going out of) the crossing set for $\ell \in\{0,+1,-1\}$. These measures have traces on $S$ that can be identified to the measures $\nu_{i n / o u t}^{\ell}$. The link is then given by (5). This point of view (developed in [13]) is more intrinsic and applies in situations where there is no trivial sets containing all the trajectories passing through the crossing. This is a real issue for general symmetric conjoint crossing.

We have reduced the dimension space to 2 but we could have assumed the dimension to be greater than 2 with a $d$-dimensionnal Laplacian and a potential $V_{P J T}\left(q_{1}, q_{2}\right)$ depending only on $q_{1}$ and $q_{2}$. Our result extends to this situation replacing $I$ by $\left\{\left(p_{1}, p_{2}\right) \wedge\left(q_{1}, q_{2}\right)=0\right\}$ and $p$ by $\left(p_{1}, p_{2}\right)$ in the non-degeneracy condition (4) and in the transition coefficient (6). It also extends to the situation where the Laplacian is replaced by a Fourier multiplier $A(D)$. One then need to turn $p$ into $\nabla A(p)$ in (4) and (6). In that situation, the existence of a submanifold satisfying similar properties than $I$ is non trivial.

The proof of this theorem relies on a normal form result which is the subject of Section 3. One reduces the analysis of the crossing to a scattering problem which is studied in Section 4. As mentioned before, this scattering problem has been already studied in [2] and [3]: we use a very slightly different method (see Remark 7 below). Provided these two results, the proof of Theorem 1 is performed in the last section following the method introduced in [13] (slightly modified to take into account the difficulties induced by the presence of the third mode). 
Before passing to the proof of the Theorem, we illustrate our result by some numerics. We choose a Gaussian initial data supported on the + level of the form

$$
\psi_{0}^{\varepsilon}(q)=(\varepsilon \pi)^{-1 / 2} \exp \left(-\frac{1}{2 \varepsilon}\left|q-q_{0}^{\varepsilon}\right|^{2}+\frac{i}{\varepsilon} p_{0} \cdot\left(q-q_{0}^{\varepsilon}\right)\right) e_{+}(q)
$$

where

$$
e_{+}(q) \in \operatorname{Ran}^{+}(q), \quad \varepsilon=10^{-2}, \quad p_{0}=(-1,0), q_{0}^{\varepsilon}=\sqrt{\varepsilon}\left(5, \frac{1}{2}\right) .
$$

The time evolves between $t=0$ and $t=\frac{\pi}{4}$ and we calculate an approximate value of the population on each mode:

$$
n^{\ell}(t)=\int_{\mathbf{R}^{2}}\left|\Pi^{\ell}(q) \psi^{\varepsilon}(q, t)\right|^{2} d q, \quad \ell \in\{-1,0,+1\} .
$$

Following [24] and [15], we perform a surface hopping algorithm:

- we sample the Wigner function,

- we propagate weighted points $\left(q_{s}^{+}, p_{s}^{+}, w\right)$ along the classical trajectory until the distance to the gap $\{q=0\}$ is minimal,

- at that point $\left(q_{s^{*}}^{+}, p_{s^{*}}^{+}, w\right)$ one generates new trajectories for each mode transporting part of the weight $w$ :

$$
\left(q_{s}^{+}, p_{s}^{+},\left(1-T^{*}\right)^{2} w\right),\left(q_{s}^{-}, p_{s}^{-}, T^{* 2} w\right), \quad\left(q_{s}^{0}, p_{s}^{0}, 2 T^{*}\left(1-T^{*}\right) w\right), \quad s>s^{*}
$$

where the result of Theorem 1 motivates the choice of the transition coefficient

$$
T^{*}=\exp \left(-\frac{\pi\left|q_{s^{*}}^{0} \wedge p_{s^{*}}^{0}\right|^{2}}{2 \varepsilon\left|p_{s^{*}}^{0}\right|^{3}}\right) .
$$

Arguing as in [24], one can prove the convergence of the algorithm. No estimation on the rate is available for the moment since it requires an improvement of the normal form result which is out of reach for the moment. In order to illustrate numerically the validity of the algorithm, we calculate a reference solution based on a grid discretization and a Strang splitting scheme. This scheme is known to have a very fast rate of convergence (see [23], [15] and [16]). Then, we compare the two outputs in the figure below: the initial population which was on the + level splits after passing once through the crossing.

3. Normal forms. In this section, we first prove a microlocal normal form which holds in the phase space close to a point of the crossing set. Then, in the second subsection, we write a two-microlocal normal form which is only valid in neighborhoods of size $\sqrt{\varepsilon}$ of the hypersurface $I$.

3.1. The microlocal normal form. The phase space $\mathbf{R}^{6}$ has the structure of the cotangent space $T^{*} \mathbf{R}^{3}$. It is endowed with a symplectic structure given by the 2 -form

$$
\omega=\mathrm{d} \gamma=\mathrm{d} p \wedge \mathrm{d} q+\mathrm{d} \tau \wedge \mathrm{d} t
$$

where $\gamma$ is the Liouville 1 -form $\gamma=p \mathrm{~d} q+\tau \mathrm{d} t$. A local change of sympletic coordinates $\kappa$ is called canonical transform and one can associate with it a unitary bounded operator of $L^{2}\left(\mathbf{R}^{3}\right)$ compatible with the change of coordinates. Such an operator $K$ is called Fourier integral operator associated with $\kappa$. It satisfies Egorov's Theorem

$$
\forall a \in \mathcal{C}_{0}^{\infty}\left(\mathbf{R}^{6}\right), \quad K^{*} \mathrm{op}_{\varepsilon}(a) K=\mathrm{op}_{\varepsilon}(a \circ \kappa)+O\left(\varepsilon^{2}\right) \text { in } \mathcal{L}\left(L^{2}\left(\mathbf{R}^{3}\right)\right) .
$$



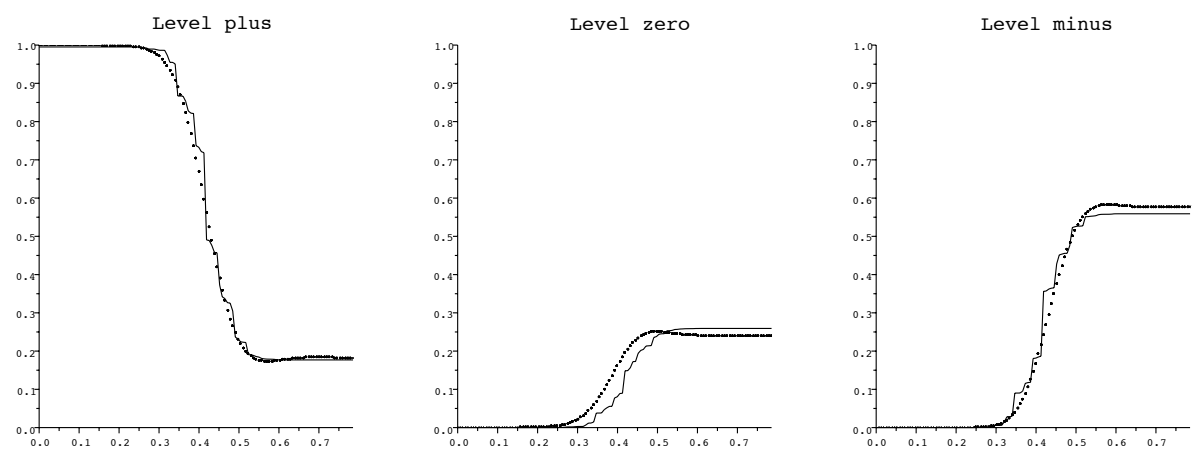

FIG. 1. Evolution of the population on each level for surface hopping algorithm (full line) and for Strang splitting scheme (dotted line).

We point out that the remainder is better than expected because we have chosen to use Weyl quantization. The reader will find a proof of this statement in [13], Section 2.2 .

We consider $\Omega$ a neighborhood of $\rho_{0}=\left(q_{0}, t_{0}, p_{0}, \tau_{0}\right) \in S$ with $p_{0} \neq 0$ and we suppose $\Omega$ small enough so that $p \neq 0$ in $\Omega$. Then, for $\ell \in\{0,+1,-1\}$, we denote by $J_{i n}^{\ell}$ (resp. $J_{\text {out }}^{\ell}$ ) the sets consisting on all the classical trajectories for the mode $\ell$ entering in (resp. ougoing of) $S \cap \Omega$. We point out that by the property of continuation of the trajectories, the sets $\left(J_{\text {in }}^{ \pm} \cup J_{\text {out }}^{\mp}\right) \cap \Omega$ are submanifolds of $T^{*} \mathbf{R}^{3}$ just as $\left(J_{\text {in }}^{0} \cup J_{\text {out }}^{0}\right) \cap \Omega$ does. Our result is the following

Theorem 2. Let $\rho_{0}=\left(q_{0}, t_{0}, p_{0}, \tau_{0}\right) \in S$ be such that $p_{0} \neq 0$. Then, there exist a local canonical transform $\kappa$ from a neighborhood of $\rho_{0}$ into some neighborhood $\tilde{\Omega}$ of 0 ,

$$
\kappa:(q, t, p, \tau) \mapsto(s, z, \sigma, \zeta), \kappa\left(\rho_{0}\right)=0,
$$

a Fourier integral operator $K$ associated with $\kappa$ and an invertible matrix-valued function A such that if $\psi^{\varepsilon}$ is a family of solutions to (1) for some initial data $\psi_{0}^{\varepsilon}$ uniformly bounded in $L^{2}\left(\mathbf{R}^{2}\right)$, then

$$
v^{\varepsilon}=K^{*} \mathrm{op}_{\varepsilon}(A) \psi^{\varepsilon}
$$

satisfies for all $\phi \in \mathcal{C}_{0}^{\infty}(\tilde{\Omega})$,

$$
\mathrm{op}_{\varepsilon}(\phi) \mathrm{op}_{\varepsilon}\left(-\sigma+V_{P J T}\left(s, \alpha(\zeta, \sigma) z_{1}\right)\right) v^{\varepsilon}=O(\varepsilon)
$$

in $L^{2}\left(\mathbf{R}^{3}\right)$ where $\alpha$ is smooth and does not vanish in $\tilde{\Omega}$.

Moreover,

$$
\begin{gathered}
\kappa(I)=\left\{z_{1}=0\right\}, \\
\kappa\left(J_{\text {in }}^{ \pm}\right)=\left\{\sigma \pm s=0, \quad z_{1}=0, \quad s \leq 0\right\} \\
\kappa\left(J_{\text {out }}^{ \pm}\right)=\left\{\sigma \mp s=0, \quad z_{1}=0, \quad s \geq 0\right\} .
\end{gathered}
$$


Proof. The proof of this Theorem proceeds in two steps. First, we find the canonical transform $\kappa$. Then, we use the properties of the matrix $V_{P J T}$ to construct $A$ such that

$$
\left[A\left(\left(\tau+\frac{|p|^{2}}{2}\right) \operatorname{Id}+V_{P J T}\left(q_{1}, q_{2}\right)\right) A^{*}\right] \circ \kappa^{-1}=-\sigma \operatorname{Id}+V_{P J T}(s, z) .
$$

Then, we obtain (8) by the Egorof's Theorem (7).

First step: the canonical transform. This first step relies on the analysis of the geometry of the crossing. We crucially use Proposition 6, p.148 in [13].

Proposition 3. There exist a local canonical transform

$$
\kappa:(q, t, p, \tau) \mapsto(s, z, \sigma, \zeta)
$$

and non-vanishing smooth functions $\lambda$ and $\mu$ such that

$$
\left\{\begin{array}{l}
\sigma=\lambda(p, \tau)\left(\tau+\frac{|p|^{2}}{2}\right), \\
s=\lambda(p, \tau) \frac{p}{|p|} \cdot q, \\
z_{1}=\lambda(p, \tau) \mu(p, \tau) \frac{p}{|p|} \wedge q .
\end{array}\right.
$$

Besides, one can choose $\zeta=\zeta(p, \tau)$ such that $(p, \tau) \mapsto(\zeta, \sigma)$ is a diffeomorphism and

$$
\lambda(p, \tau)_{\mid S}=|p|^{-1 / 2} .
$$

Let us shortly describe the proof of Proposition 6 in [13]. Observing that

$$
\left\{\tau+\frac{|p|^{2}}{2}, \frac{p}{|p|} \cdot q\right\}=|p|
$$

we obtain the existence of $\lambda$ such that $s$ and $\sigma$ satisfy the bracket condition $\{\sigma, s\}=1$. Therefore we necessarily have (13). Besides, for any function $\mu=\mu(p, \tau)$, one has

$$
\left\{\lambda(p, \tau)\left(\tau+\frac{|p|^{2}}{2}\right), \lambda(p, \tau) \mu(p, \tau) \frac{p}{|p|} \wedge q\right\}=0 .
$$

Thus, it is enough to find $\mu$ such that

$$
\left\{\lambda(p, \tau) \frac{p}{|p|} \cdot q, \lambda(p, \tau) \mu(p, \tau) \frac{p}{|p|} \wedge q\right\}=0 .
$$

Once $\mu$ is built, one completes the symplectic system $\left(\sigma, s, z_{1}\right)$ by Darboux Theorem.

By the definition of $S$ and $I$, one has $\kappa(S)=\left\{\sigma=s=z_{1}=0\right\}$ and $\kappa(I)=\left\{z_{1}=0\right\}$. Moreover, since $\lambda \neq 0$ (since $p \neq 0$ in $\Omega$ ), we have $\kappa(\Sigma)=\left\{\sigma\left(\sigma^{2}-s^{2}-z_{1}^{2}\right)=0\right\}$. In order to find the precise equations of $\kappa\left(J_{\text {in }}^{ \pm}\right)$and $\kappa\left(J_{\text {out }}^{ \pm}\right)$, we observe that

$$
\kappa\left(J_{\text {in } / \text { out }}^{ \pm}\right) \subset\left\{\sigma^{2}=s^{2}, z_{1}=0\right\}
$$

and we consider the Hamiltonian vector fields $H_{\lambda^{ \pm}}$. We have outside $S$

$$
H_{\lambda^{ \pm}}=\partial_{t}+p \cdot \nabla_{q} \mp \frac{q}{|q|} \cdot \nabla_{p}
$$


Observing that if the trajectory $\left(q_{s}^{ \pm}, p_{s}^{ \pm}\right)$passes through $S$ at $s=0$ we have

$$
q_{s}^{ \pm}=s p_{0}+o(s)
$$

we deduce

$$
H_{\lambda^{ \pm}}\left(\rho_{s}^{ \pm}\right)=\partial_{t}+p \cdot \nabla_{q} \mp \operatorname{sgn}(s) \frac{p_{0}}{\left|p_{0}\right|} \cdot \nabla+o(1) .
$$

By the definition of $\sigma$ and $s$, we have

$$
H_{\sigma \pm s}=\lambda\left[\partial_{t}+p \cdot \nabla_{q} \pm \frac{p}{|p|} \cdot \nabla\right] \text { on } S .
$$

Therefore, we obtain

$$
H_{\lambda+}\left(\rho_{s}^{+}\right) \underset{s \rightarrow 0^{-}}{\longrightarrow} \partial_{t}+p_{0} \cdot \nabla_{q}+\frac{p_{0}}{\left|p_{0}\right|} \cdot \nabla_{q}=\lambda^{-1} H_{\sigma+s} .
$$

This equation implies that $\kappa\left(J_{i n}^{+}\right) \subset\{\sigma+s=0\}$, whence the equation of $\kappa\left(J_{i n}^{+}\right)$by (14) and dimension considerations. One argues similarly for $J_{\text {out }}^{+}, J_{\text {in }}^{-}$and $J_{o u t}^{-}$.

Second step: the gauge transform. We now transform the symbol $\left(\tau+\frac{|p|^{2}}{2}\right) \operatorname{Id}+$ $V(q)$ in order to see the coordinates $(s, z, \sigma, \zeta)$ in the symbol. We first use the matrix

$$
M=\frac{1}{\sqrt{2}}\left(\begin{array}{ccc}
1 & 1 & 0 \\
1 & -1 & 0 \\
0 & 0 & \sqrt{2}
\end{array}\right)
$$

and observe that $M=M^{*}=M^{-1}$ and $M V(q) M=W(q)$ with

$$
W(q)=\left(\begin{array}{ccc}
0 & q_{1} & q_{2} \\
q_{1} & 0 & 0 \\
q_{2} & 0 & 0
\end{array}\right)
$$

The matrix $M$ allows to transform a system with a matrix-valued potential $V$ into a system with the potential $W$ which is easier to manipulate since most of its coefficients are zero. We then use the following lemma which comes from a straightforward computation.

Lemma 4. Consider the matrix $R(p)=\frac{1}{|p|}\left(\begin{array}{ccc}|p| & 0 & 0 \\ 0 & p_{1} & -p_{2} \\ 0 & p_{2} & p_{1}\end{array}\right)$ then $R(p)$ is invertible for $p \neq 0$ and we have

$$
R(p) W(q) R(p)^{*}=W\left(\frac{p}{|p|} \cdot q, \frac{p}{|p|} \wedge q\right) .
$$

Note that $R$ is a matrix of rotation. We can now conclude the proof of Theorem 2 . Set

$$
P(q, p, \tau)=\left(\tau+\frac{|p|^{2}}{2}\right) \mathrm{Id}-V(q) \text { and } \tilde{R}(p)=M R(p) M
$$


We have

$$
\tilde{R}(p) P(q, p, \tau) \tilde{R}(p)^{*}=\left(\tau+\frac{|p|^{2}}{2}\right) \operatorname{Id}-V\left(\frac{p}{|p|} \cdot q, \frac{p}{|p|} \wedge q\right) .
$$

Therefore, using the canonical transform of Proposition 3, we have

$$
\left[\lambda(p, \tau) \tilde{R}(p) P(q, p, \tau) \tilde{R}(p)^{*}\right] \circ \kappa^{-1}(s, z, \sigma, \zeta)=\sigma \operatorname{Id}-V\left(s, \alpha(p, \tau) z_{1}\right) .
$$

Let us consider now a Fourier integral operator $U$ associated with $\kappa$ and set

$$
A(p, \tau)=\lambda(p, \tau)^{1 / 2} \tilde{R}(p) .
$$

We have

$$
U \mathrm{op}_{\varepsilon}(A) \mathrm{op}_{\varepsilon}(P) \mathrm{op}_{\varepsilon}\left(A^{*}\right) U^{*}=\mathrm{op}_{\varepsilon}\left(\sigma \mathrm{Id}-V\left(s, \alpha(\sigma, \zeta) z_{1}\right)\right)+O(\varepsilon)
$$

in $\mathcal{L}\left(L^{2}\left(\mathbf{R}^{3}\right)\right)$.

3.2. The 2-microlocal normal form. In this section, we want to ameliorate the normal form in open sets localized at a distance of order $\sqrt{\varepsilon}$ of the hypersurface I. For simplicity, we set

$$
J=\left(\begin{array}{ccc}
1 & 0 & 0 \\
0 & -1 & 0 \\
0 & 0 & 0
\end{array}\right), \quad L=\frac{1}{\sqrt{2}}\left(\begin{array}{ccc}
0 & 0 & 1 \\
0 & 0 & 1 \\
1 & 1 & 0
\end{array}\right)
$$

We shall take advantage of the fact that if $a \in \mathcal{C}_{0}^{\infty}\left(\mathbf{R}^{7}\right)$,

$$
\mathrm{op}_{\varepsilon}{ }^{I}(a) \mathrm{op}_{\varepsilon}\left(-\sigma \operatorname{Id}+V_{P J T}\left(s, \alpha(\sigma, \zeta) z_{1}\right)\right)=\mathrm{op}_{\varepsilon}{ }^{I}(a) \mathrm{op}_{\varepsilon}{ }^{I}\left(-\sigma \operatorname{Id}+V_{P J T}(s, \alpha(\sigma, \zeta) \sqrt{\varepsilon} \eta)\right) \text {. }
$$

We set

$$
\begin{aligned}
Q & =-\sigma \mathrm{Id}+s J+\sqrt{\varepsilon} \alpha(\sigma, \zeta) \eta L, \\
Q_{0} & =-\sigma \mathrm{Id}+s J+\sqrt{\varepsilon} \alpha(0, \zeta) \eta L .
\end{aligned}
$$

We prove the following result.

Proposition 5. For every ball B of $\mathbf{R}_{\eta}$, there exist smooth matrix-valued functions $C$ and $\tilde{C}$ such that for all $a \in \mathcal{C}_{0}^{\infty}\left(\mathbf{R}^{6} \times B\right)$,

$$
\left\|\operatorname{op}_{\varepsilon}{ }^{I}(a)\left[\mathrm{op}_{\varepsilon}{ }^{I}(\mathrm{Id}+\sqrt{\varepsilon} C) \mathrm{op}_{\varepsilon}(Q)-\mathrm{op}_{\varepsilon}\left(Q_{0}\right) \mathrm{op}_{\varepsilon}{ }^{I}(\mathrm{Id}+\sqrt{\varepsilon} \tilde{C})\right]\right\|_{\mathcal{L}\left(L^{2}\right)}=O(\varepsilon) .
$$

Proof. We want to realize

$$
\mathrm{op}_{\varepsilon}{ }^{I}(\mathrm{Id}+\sqrt{\varepsilon} C) \mathrm{op}_{\varepsilon}(Q)=\mathrm{op}_{\varepsilon}\left(Q_{0}\right) \mathrm{op}_{\varepsilon}^{I}(\mathrm{Id}+\sqrt{\varepsilon} \tilde{C})+O(\varepsilon) .
$$

For this, we successively consider the terms of order 1 and $\sqrt{\varepsilon}$ in the development of the preceeding equality by symbolic calculus. The terms of order 0 are equal and the equality for the terms of order $\sqrt{\varepsilon}$ reads

$$
\alpha(\sigma, \zeta) \eta L-\alpha(0, \zeta) \eta L+\sigma(\tilde{C}-C)+s(C J-J \tilde{C})=0 .
$$


We choose $\tilde{C}=-{ }^{t} C$ with

$$
C=f\left(\begin{array}{lll}
0 & 0 & 1 \\
0 & 0 & 1 \\
0 & 0 & 0
\end{array}\right)
$$

so that the second equation writes in view of $C J=0$ and $J \tilde{C}=-{ }^{t}(C J)=0$

$$
[\eta(\alpha(\sigma, \zeta)-\alpha(0, \zeta))-\sqrt{2} \sigma f] L=0
$$

Hence

$$
f(\sigma, z, \eta)=\frac{\eta}{\sqrt{2}} \frac{\alpha(\sigma, \zeta)-\alpha(0, \zeta)}{\sigma}
$$

This closes the proof of the Proposition.

4. Scattering matrix for the pseudo Jahn-Teller model. In this section, we analyze the differential system

$$
-i \partial_{s}\left(\begin{array}{c}
v_{+} \\
v_{-} \\
v_{0}
\end{array}\right)=\left(\begin{array}{ccc}
s & 0 & z / \sqrt{2} \\
0 & -s & \bar{z} / \sqrt{2} \\
\bar{z} / \sqrt{2} & z / \sqrt{2} & 0
\end{array}\right)\left(\begin{array}{c}
v_{+} \\
v_{-} \\
v_{0}
\end{array}\right)=V_{P J T}(s, z)\left(\begin{array}{c}
v_{+} \\
v_{-} \\
v_{0}
\end{array}\right)
$$

where $z$ is a non-zero complex parameter. In the following, we will only use the case of real-valued parameters $z$, however, it is interesting to see that the complex-valued situation can be treated. We prove the following scattering result.

Theorem 6. There exist $\left(\alpha^{-}, \alpha^{+}, \alpha^{0}\right)$ and $\left(\omega^{-}, \omega^{+}, \omega^{0}\right)$ such that

$$
\begin{aligned}
& v_{\ell}(s)=\exp \left[\ell i\left(\frac{s^{2}}{2}+\frac{|z|^{2}}{2} \ln |s|\right)\right] \alpha^{\ell}+o(1) \text { as } s \rightarrow-\infty, \\
& v_{\ell}(s)=\exp \left[\ell i\left(\frac{s^{2}}{2}+\frac{|z|^{2}}{2} \ln |s|\right)\right] \omega^{\ell}+o(1) \text { as } s \rightarrow+\infty .
\end{aligned}
$$

Besides

$$
\left(\begin{array}{c}
\omega^{+} \\
\omega^{-} \\
\omega^{0}
\end{array}\right)=S(z)\left(\begin{array}{c}
\alpha^{+} \\
\alpha^{-} \\
\alpha^{0}
\end{array}\right)
$$

with

$$
S(z)=\left(\begin{array}{ccc}
e^{-\pi|z|^{2} / 2} & i \Omega(z)^{2}\left[1-e^{-\pi|z|^{2} / 2}\right] & \sqrt{2} e^{i \pi / 4} \Omega(z) \theta(z) \\
-i \overline{\Omega(z)}^{2}\left[1-e^{-\pi|z|^{2} / 2}\right] & e^{-\pi|z|^{2} / 2} & -\sqrt{2} e^{-i \pi / 4} \overline{\Omega(z)} \theta(z) \\
-\sqrt{2} e^{-i \pi / 4} \overline{\Omega(z)} \theta(z) & \sqrt{2} e^{i \pi / 4} \Omega(z) \theta(z) & 2 e^{-\pi|z|^{2} / 2}-1
\end{array}\right)
$$

and

$$
\Omega(z)=\frac{z}{|z|} \frac{\Gamma\left(1-i \frac{z^{2}}{4}\right)}{\left|\Gamma\left(1-i \frac{z^{2}}{4}\right)\right|}, \quad \theta(z)=\sqrt{1-e^{-\pi z^{2} / 2}} e^{-\pi z^{2} / 4} .
$$

Note that the indices,+- and 0 are not directly connected with the modes $\lambda^{\ell}$. However their connection will be clarified in Section 5.2. 
4.1. Providing two independent solutions with contour integrals. Classical theorems give that the solutions form a tridimensional linear space of analytic functions. The parity block decomposition of $V_{P J T}(s, z)$ induces that the space of solutions splits into two subspaces: a bidimensional one with $v_{ \pm}$even and $v_{0}$ odd, and a unidimensional one with $v_{ \pm}$odd and $v_{0}$ even. For an even (respectively odd) analytic function $v$, there exists an analytic function $f$ such that $v(s)=f\left(s^{2} / 2\right)$ (respectively $\left.v(s)=s f\left(s^{2} / 2\right)\right)$. Hence the system, in the case of the bidimensional subspace,

$$
-i\left(\begin{array}{ccc}
\partial_{t} & 0 & 0 \\
0 & \partial_{t} & 0 \\
0 & 0 & 1+2 t \partial_{t}
\end{array}\right)\left(\begin{array}{c}
f_{+} \\
f_{-} \\
f_{0}
\end{array}\right)=V_{P J T}(1, z)\left(\begin{array}{c}
f_{+} \\
f_{-} \\
f_{0}
\end{array}\right) .
$$

To solve (19), we will use the method of contour integral (see for instance [6] Chap. $\mathrm{XV})$ : we look for solutions of the form

$$
\left(\begin{array}{c}
f_{+}(t) \\
f_{-}(t) \\
f_{0}(t)
\end{array}\right)=\int_{\Lambda} e^{i t \tau}\left(\begin{array}{c}
F_{+}(\tau) \\
F_{-}(\tau) \\
F_{0}(\tau)
\end{array}\right) d \tau
$$

where $\Lambda$ is an oriented path of $\mathbf{C}$ and the $F_{*}$ 's are functions holomorphic in a neighbourhood of $\Lambda$. Injecting (20) into (19) we get

$$
-\int_{\Lambda} e^{i t \tau} i\left(\begin{array}{ccc}
i \tau & 0 & 0 \\
0 & i \tau & 0 \\
0 & 0 & 1+2 i t \tau
\end{array}\right)\left(\begin{array}{c}
F_{+}(\tau) \\
F_{-}(\tau) \\
F_{0}(\tau)
\end{array}\right) d \tau=\int_{\Lambda} e^{i t \tau} V_{P J T}(1, z)\left(\begin{array}{c}
F_{+}(\tau) \\
F_{-}(\tau) \\
F_{0}(\tau)
\end{array}\right) d \tau
$$

and after integrating by parts

$$
\begin{aligned}
{\left[e^{i t \tau}\left(\begin{array}{c}
0 \\
0 \\
-2 i \tau F_{0}(\tau)
\end{array}\right)\right]_{\Lambda}+\int_{\Lambda} e^{i t \tau}\left(\begin{array}{c}
0 \\
0 \\
2 i\left[\tau F_{0}^{\prime}(\tau)+F_{0}(\tau)\right]
\end{array}\right) d \tau } \\
\quad=\int_{\Lambda} e^{i t \tau}\left(\begin{array}{ccc}
-\tau+1 & 0 & \frac{z}{\sqrt{2}} \\
0 & -\tau-1 & \frac{\bar{z}}{\sqrt{2}} \\
\frac{\bar{z}}{\sqrt{2}} & \frac{z}{\sqrt{2}} & i
\end{array}\right)\left(\begin{array}{c}
F_{+}(\tau) \\
F_{-}(\tau) \\
F_{0}(\tau)
\end{array}\right) d \tau .
\end{aligned}
$$

Hence, it is enough to choose the $F_{*}$ 's such that

$$
\left(\begin{array}{c}
0 \\
0 \\
2 i \tau F_{0}^{\prime}(\tau)
\end{array}\right)=\left(\begin{array}{ccc}
-\tau+1 & 0 & \frac{z}{\sqrt{2}} \\
0 & -\tau-1 & \frac{z}{\sqrt{2}} \\
\frac{\bar{z}}{\sqrt{2}} & \frac{z}{\sqrt{2}} & -i
\end{array}\right)\left(\begin{array}{c}
F_{+}(\tau) \\
F_{-}(\tau) \\
F_{0}(\tau)
\end{array}\right)
$$

and $\Lambda$ such that

$$
\left[e^{i t \tau} \tau F_{0}(\tau)\right]_{\Lambda}=0
$$

The system is exactly solvable and gives, up to a global constant,

$$
F_{0}(\tau)=\frac{e^{i \frac{|z|^{2}}{4}[\ln (\tau+1)-\ln (\tau-1)]}}{\sqrt{\tau}}, \quad F_{+}(\tau)=\frac{z}{\sqrt{2}} \frac{F_{0}(\tau)}{\tau-1}, \quad F_{-}(\tau)=\frac{\bar{z}}{\sqrt{2}} \frac{F_{0}(\tau)}{\tau+1} .
$$

Now $F_{*}(\tau)$ has three singularities, namely $\pm 1,0$ and $F_{*}(\tau)$ is multivalued. More explicitly, considering a closed contour, the value of $F_{*}(\tau)$ is changed according to the 
following rule: if the contour goes $n_{ \pm}$times anticlockwise around \pm 1 then $\ln (\tau \mp 1)$ is changed into $\ln (\tau \mp 1)+i 2 \pi n_{ \pm}$and if it goes $n_{0}$ times anticlockwise around 0 then $\sqrt{\tau}$ is changed into $(-1)^{n_{0}} \sqrt{\tau}$. If we choose a closed contour $\Lambda$ that avoids the singularities of $F_{*}$ and solves (21), the corresponding function (20) is then well defined and is a solution of (19), the only thing that one has to check is that it does provide a non trivial solution. We will focus on the following contours: they enclose only the singularities \pm 1 and go once around each of them. There are exacly two of them depending on whether we stay below or above the singularity 0 (in the first case the contour has the shape of a $\cup$ and the second of a $\cap$ ). The idea is now to use residue arguments to get explicit formulas for the contribution of the different singularities. Multiplying by $-\sqrt{2} /|z|$, we define our two solutions to be

$$
\left(\begin{array}{c}
v_{+}(s) \\
v_{-}(s) \\
v_{0}(s)
\end{array}\right)=\int_{\Lambda} e^{i \frac{s^{2}}{2} \tau} e^{i \frac{|z|^{2}}{4}[\ln (\tau+1)-\ln (\tau-1)]}\left(\begin{array}{c}
\frac{z}{|z|} \frac{1}{\tau-1} \\
\frac{\bar{z}}{|z|} \frac{1}{\tau+1} \\
\frac{\sqrt{2}}{|z|} s
\end{array}\right) \frac{d \tau}{\sqrt{\tau}}
$$

where the $\Lambda^{\cup}$ and $\Lambda^{\cap}$ contours are decomposed according to Figure 2 and the determination of the two logarithms and the square root is such that it coincides with the usual definition (for positive real numbers) at the point $M$.
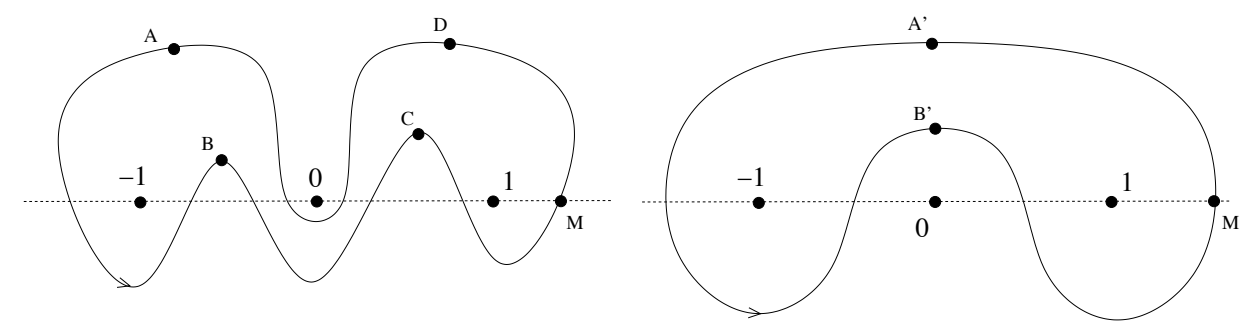

FIG. 2. decomposition of the $\Lambda^{\cup}$ and $\Lambda^{\cap}$ contours

For the sake of clarity, the points $A, B, C$ and $D$ (respectively $A^{\prime}$ and $B^{\prime}$ ) have been split but they have to be thought of as the same geometric point.

REMARK 7. It is in the choice of these contours that our proof differ of the proof of [2] and [3].

To study separately the different pieces, we will first show the following residue results.

LEMMA 8. Let $\beta$ be a non zero real number, $w(\tau)$ an holomorphic function of $\tau$ in a convex open neighborhood $\Omega$ of a point $\alpha$ of the real axis and $\Lambda$ an oriented path of $\Omega$ with both ends in the upper half plane. Then, in the limit $|s| \rightarrow+\infty$

1. if $\Lambda$ does not surround $\alpha$,

$$
\int_{\Lambda} e^{i \frac{s^{2}}{2} \tau} e^{i \beta \ln (\tau-\alpha)} w(\tau) d \tau=O\left(e^{-\frac{s^{2}}{2} \delta}\right), \quad \int_{\Lambda} e^{i \frac{s^{2}}{2} \tau} w(\tau) \frac{d \tau}{\sqrt{\tau-\alpha}}=O\left(e^{-\frac{s^{2}}{2} \delta}\right)
$$

where $\delta$ is the minimum of the imaginary part of the two endpoints;

2. if $\Lambda$ does surround $\alpha$ once and anticlockwise,

$$
\begin{aligned}
\int_{\Lambda} e^{i \frac{s^{2}}{2} \tau} e^{i \beta \ln (\tau-\alpha)} w(\tau) d \tau= & -4 i e^{i \beta \ln 2} e^{\pi \beta / 2} \sinh (\pi \beta) \Gamma(1+i \beta) w(\alpha) \\
& \times \frac{e^{i\left[\frac{s^{2}}{2} \alpha-2 \beta \ln |s|\right]}}{s^{2}}\left[1+O\left(\frac{1}{s^{2}}\right)\right]
\end{aligned}
$$




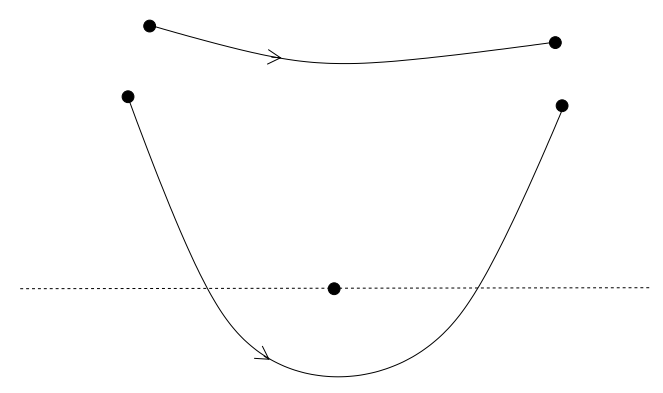

FIG. 3. non-surrounding and surrounding contours

$$
\int_{\Lambda} e^{i \frac{s^{2}}{2} \tau} w(\tau) \frac{d \tau}{\sqrt{\tau-\alpha}}=2 \sqrt{2 \pi} e^{i \pi / 4} w(\alpha) \frac{e^{i \frac{s^{2}}{2} \alpha}}{|s|}\left[1+O\left(\frac{1}{s^{2}}\right)\right]
$$

where the determination of the logarithm and of the square root is such that it coincides with the usual definition for positive real numbers. Furthermore (23) is locally uniform in $\beta$.

Proof.

1. It follows from a crude estimate of the term $e^{i \frac{s^{2}}{2} \tau}$ for the straight line path.

2. We deform the contour into the one presented in Figure 4 where the two vertical lines have been split for a better understanding i.e. $B$ and $E$ (respectively $C$ and $D)$ are in fact the same geometric point. The radius of the small circle is chosen to be $\lambda(s) / s^{2}$ where $\lambda(s) \rightarrow 0$ as $|s| \rightarrow+\infty$.

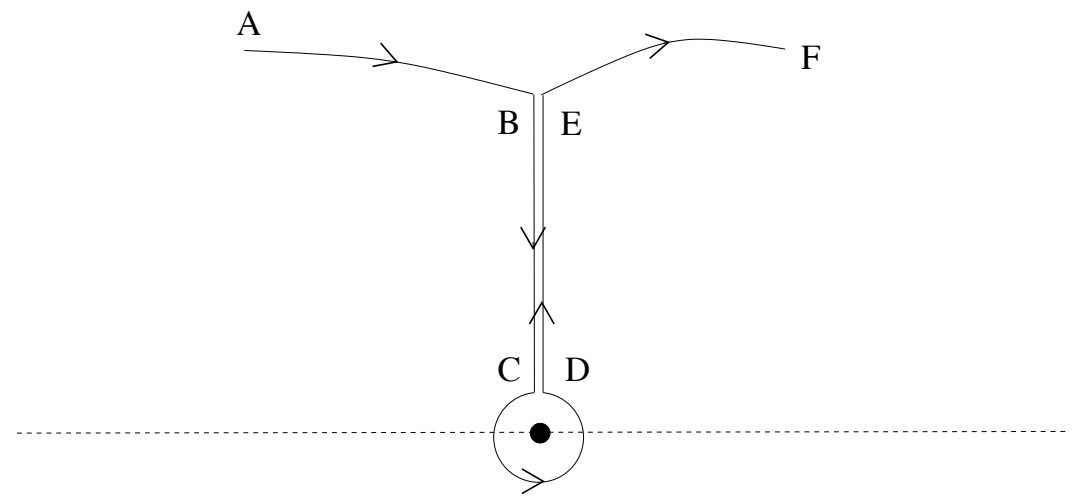

FIG. 4. deformation of the surrounding contour

The pieces $A B$ and $E F$ are exponentially small by the first point. The piece $B C$ is $-e^{2 \pi \beta}$ times (respectively equal to) the piece $D E$ for the logarithm (respectively the square root). Let us compute the piece $C D$ parameterizing 
the circle by $\alpha+\frac{\lambda(s)}{s^{2}} e^{i \theta}$ for $-3 \pi / 2 \leq \theta \leq \pi / 2$ :

$$
\begin{aligned}
& \int_{C}^{D} e^{i \frac{s^{2}}{2} \tau} e^{i \beta \ln (\tau-\alpha)} w(\tau) d \tau \\
& =\frac{\lambda(s)}{s^{2}} e^{i \frac{s^{2}}{2} \alpha} e^{-2 i \beta \ln |s|} e^{i \beta \ln \lambda(s)} \int_{-3 \pi / 2}^{\pi / 2} e^{i \frac{\lambda(s)}{2} e^{i \theta}} e^{-\beta \theta} w\left(\alpha+\frac{\lambda(s)}{s^{2}} e^{i \theta}\right) e^{i \theta} i d \theta \\
& =O\left(\frac{\lambda(s)}{s^{2}}\right) \text {, }
\end{aligned}
$$

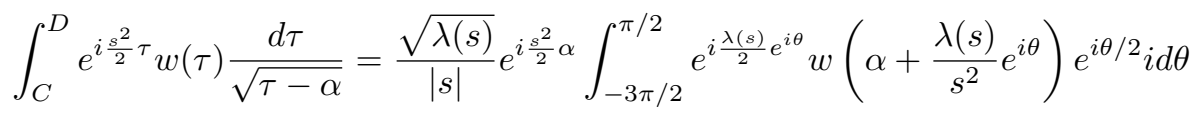

$$
\begin{aligned}
& =O\left(\frac{\sqrt{\lambda(s)}}{|s|}\right) \text {. }
\end{aligned}
$$

Finally, we compute the contribution of the piece $D E$ parameterizing the vertical segment by $\alpha+2 \frac{i}{s^{2}} u$ for $\lambda(s) / 2 \leq u \leq s^{2} \delta / 2$ :

$$
\begin{aligned}
& \int_{D}^{E} e^{i \frac{s^{2}}{2} \tau} e^{i \beta \ln (\tau-\alpha)} w(\tau) d \tau \\
= & \frac{e^{i \frac{s^{2}}{2} \alpha} e^{-2 i \beta \ln |s|}}{s^{2}} e^{i \beta \ln 2} e^{-\pi \beta / 2} 2 i \int_{\lambda(s) / 2}^{s^{2} \delta / 2} e^{-u} u^{i \beta} w\left(\alpha+2 \frac{i}{s^{2}} u\right) d u \\
= & \frac{e^{i \frac{s^{2}}{2} \alpha} e^{-2 i \beta \ln |s|}}{s^{2}} e^{i \beta \ln 2} e^{-\pi \beta / 2} 2 i\left[w(\alpha) \int_{0}^{+\infty} e^{-u} u^{i \beta} d u+O\left(\lambda(s)+\frac{1}{s^{2}}\right)\right] \\
= & \frac{e^{i \frac{s^{2}}{2} \alpha} e^{-2 i \beta \ln |s|}}{s^{2}} e^{i \beta \ln 2} e^{-\pi \beta / 2} 2 i \Gamma(1+i \beta) w(\alpha)+O\left(\frac{\lambda(s)}{s^{2}}+\frac{1}{s^{4}}\right), \\
= & \frac{e^{i \frac{s^{2}}{2}} \alpha}{|s|} \sqrt{2} e^{i \pi / 4} \int_{\lambda(s) / 2}^{s^{2} \delta / 2} e^{-u} u^{-1 / 2} w\left(\alpha+2 \frac{i}{s^{2}} u\right) d u \\
= & \frac{e^{i \frac{s^{2}}{2}} \alpha}{|s|} \sqrt{2} e^{i \pi / 4}\left[w(\alpha) \int_{0}^{+\infty} e^{-u} u^{-1 / 2} d u+O\left(\sqrt{\lambda(s)}+\frac{1}{s^{2}}\right)\right] \\
= & \frac{e^{i \frac{s^{2}}{2}} \alpha}{|s|} \sqrt{2} e^{i \pi / 4} w(\alpha) \Gamma\left(\frac{1}{2}\right)+O\left(\frac{\sqrt{\lambda(s)}}{|s|}+\frac{1}{|s|^{3}}\right) .
\end{aligned}
$$

The result then follows from the choice $\lambda(s)=1 / s^{2}\left(\right.$ respectively $\left.\lambda(s)=1 / s^{4}\right)$ for the logarithm (respectively the square root) and the fact that $\Gamma(1 / 2)=$ $\sqrt{\pi}$.

Let us now give explicitly the contributions in the case of the $\Lambda^{\cap}$ contour pro- 
ceeding with integration by parts and several applications of formula (23).

$$
\begin{aligned}
& v_{+}^{\cap}(s)=2 i \frac{z}{|z|} e^{-\pi|z|^{2} / 8} \frac{\sinh \left(\pi|z|^{2} / 4\right)}{|z|^{2} / 4} \Gamma\left(1-i \frac{|z|^{2}}{4}\right) e^{i\left[\frac{s^{2}}{2}+\frac{|z|^{2}}{2} \ln |s|\right]}+O\left(\frac{1}{s^{2}}\right) \\
& v_{-}^{\cap}(s)=2 \frac{\bar{z}}{|z|} e^{-\pi|z|^{2} / 8} \frac{\sinh \left(\pi|z|^{2} / 4\right)}{|z|^{2} / 4} \Gamma\left(1+i \frac{|z|^{2}}{4}\right) e^{-i\left[\frac{s^{2}}{2}+\frac{|z|^{2}}{2} \ln |s|\right]}+O\left(\frac{1}{s^{2}}\right) \\
& v_{0}^{\cap}(s)=O\left(\frac{1}{|s|}\right) .
\end{aligned}
$$

The asymptotics for the $\Lambda^{\cup}$ contour can be mostly deduce from the following considerations (up to exponentially small errors):

- the contribution of the piece $C D$ is the same as $B^{\prime} A^{\prime}$ whereas the contribution of $A B$ is the opposite of $A^{\prime} B^{\prime}$ (as we have looped once around the origin, the square root is changed into its opposite),

- the contribution of $D A$ is $-e^{\pi|z|^{2} / 2}$ times the contribution of $B C$ (as we have looped once around $1, \ln (\tau-1)$ is translated by $2 i \pi$ and the orientation of the path is reversed),

- in the case of $v_{ \pm}(s)$, the contribution of $B C$ is $O(1 /|s|)$ whereas in the case of $v_{0}(s)$ it is $O(1)$ and given by $(24)$.

(28) $v_{+}^{\cup}(s)=-2 i \frac{z}{|z|} e^{-\pi|z|^{2} / 8} \frac{\sinh \left(\pi|z|^{2} / 4\right)}{|z|^{2} / 4} \Gamma\left(1-i \frac{|z|^{2}}{4}\right) e^{i\left[\frac{s^{2}}{2}+\frac{|z|^{2}}{2} \ln |s|\right]}+O\left(\frac{1}{|s|}\right)$

(29) $v_{-}^{\cup}(s)=2 \frac{\bar{z}}{|z|} e^{-\pi|z|^{2} / 8} \frac{\sinh \left(\pi|z|^{2} / 4\right)}{|z|^{2} / 4} \Gamma\left(1+i \frac{|z|^{2}}{4}\right) e^{-i\left[\frac{s^{2}}{2}+\frac{|z|^{2}}{2} \ln |s|\right]}+O\left(\frac{1}{|s|}\right)$

(30) $v_{0}^{\cup}(s)=\operatorname{sgn}(s) \frac{8}{|z|} e^{i \pi / 4} \sqrt{\pi} \sinh \left(\frac{\pi|z|^{2}}{4}\right)+O\left(\frac{1}{|s|}\right)$.

For later use, we introduce the functions

$$
\begin{gathered}
a(z)=2 \frac{z}{|z|} e^{-\pi|z|^{2} / 8} \frac{\sinh \left(\pi|z|^{2} / 4\right)}{|z|^{2} / 4} \Gamma\left(1-i \frac{|z|^{2}}{4}\right), \quad b(z)=\frac{8}{|z|} e^{i \pi / 4} \sqrt{\pi} \sinh \left(\frac{\pi|z|^{2}}{4}\right) \\
\varphi(s, z)=\frac{s^{2}}{2}+\frac{|z|^{2}}{2} \ln |s| .
\end{gathered}
$$

Using that for a real number $u$ we have $\overline{\Gamma(1+i u)}=\Gamma(1-i u)$ and $|\Gamma(1+i u)|^{2}=$ $\frac{\pi u}{\sinh (\pi u)}$, we get the useful relations

$$
|b|^{2}=\left(2 e^{\pi|z|^{2} / 2}-2\right)|a|^{2}, \quad \sqrt{2|a|^{2}+|b|^{2}}=\sqrt{2} e^{\pi|z|^{2} / 4}|a| .
$$

4.2. The third solution. As an application of Theorem 12 of the Appendix, let $V^{\cup}(s)$ and $V^{\cap}(s)$ denote the two independent solutions built in the preceding section through formula (22) and the corresponding $\Lambda^{\cup}$ and $\Lambda^{\cap}$ contours represented in Figure 2. We set

$$
V^{\cup \cap}(s):=\frac{V^{\cup}(s)}{\left\|V^{\cup}(s)\right\|} \wedge \frac{V^{\cap}(s)}{\left\|V^{\cap}(s)\right\|}
$$


and it follows from the preceding result that $V^{\cup \cap}(s)$ is a solution of (18). Using (44), the asymptotics of our three (orthonormal!) solutions are

$$
\begin{gathered}
\frac{V^{\cup}(s)}{\left\|V^{\cup}(s)\right\|}=\frac{\sqrt{2} e^{-\pi|z|^{2} / 4}}{2|a|}\left(\begin{array}{c}
-i a e^{i \varphi} \\
\bar{a} e^{-i \varphi} \\
\operatorname{sgn}(s) b
\end{array}\right)+O\left(\frac{1}{|s|}\right), \\
\frac{V^{\cap}(s)}{\left\|V^{\cap}(s)\right\|}=\frac{\sqrt{2}}{2|a|}\left(\begin{array}{c}
i a e^{i \varphi} \\
\bar{a} e^{-i \varphi} \\
0
\end{array}\right)+O\left(\frac{1}{|s|}\right), \\
V^{\cup \cap}(s)=\frac{e^{-\pi|z|^{2} / 4}}{2|a|^{2}}\left(\begin{array}{c}
-\operatorname{sgn}(s) a \bar{b} \bar{b} e^{i \varphi} \\
-\operatorname{sgn}(s) i \bar{a} \bar{b} e^{-i \varphi} \\
2 i|a|^{2}
\end{array}\right)+O\left(\frac{1}{|s|}\right) .
\end{gathered}
$$

4.3. Scattering matrix. To summarize, we have

$\lim _{s \rightarrow \pm \infty}\left(\begin{array}{ccc}e^{i\left[\frac{s^{2}}{2}+\frac{|z|^{2}}{2} \ln |s|\right]} & 0 & 0 \\ 0 & e^{-i\left[\frac{s^{2}}{2}+\frac{|z|^{2}}{2} \ln |s|\right]} & 0 \\ 0 & 0 & 1\end{array}\right)\left(\begin{array}{lll}\frac{V^{\cup}(s)}{\left\|V^{\cup}(s)\right\|} & \frac{V^{n}(s)}{\left\|V^{n}(s)\right\|} & V^{\cup n}(s)\end{array}\right)=M_{ \pm}$

where

$$
M_{ \pm}:=\left(\begin{array}{ccc}
-i \frac{\sqrt{2}}{2} \frac{a}{|a|} e^{-\pi|z|^{2} / 4} & i \frac{\sqrt{2}}{2} \frac{a}{|a|} & \mp i \frac{\sqrt{2}}{2} \frac{a}{|a|} \frac{\bar{b}}{|b|} \sqrt{1-e^{-\pi|z|^{2} / 2}} \\
\frac{\sqrt{2}}{2} \frac{a}{|a|} e^{-\pi|z|^{2} / 4} & \frac{\sqrt{2}}{2} \frac{\bar{a}}{|a|} & \mp i \frac{\sqrt{2}}{2} \frac{a}{|a|} \frac{b}{|b|} \sqrt{1-e^{-\pi|z|^{2} / 2}} \\
\pm \frac{b}{|b|} \sqrt{1-e^{-\pi|z|^{2} / 2}} & 0 & i e^{-\pi|z|^{2} / 4}
\end{array}\right)
$$

and the scattering matrix is given by $S=M_{+} M_{-}^{-1}$ which closes the proof of Theorem 6 .

5. Two-scale Wigner measure and Pseudo Jahn-Teller Hamiltonians. In this section, we shall prove Theorem 1. We proceed in two steps: we first prove the propagation of the two-scale Wigner measure at infinity, then we calculate the transfer's rates at finite distance thanks to the scattering result of the preceding section. We shall work in the coordinates $(s, z, \sigma, \zeta)$ introduced in Section 3 and then translate the result in the original coordinates $(q, t, p, \tau)$. For this reason, we consider the family $\left(v^{\varepsilon}\right)$ of Theorem 2 . It satisfies microlocally in $\tilde{\Omega}$,

$$
\mathrm{op}_{\varepsilon}(Q) v^{\varepsilon}=\varepsilon f^{\varepsilon},
$$

where for any $\chi \in \mathcal{C}_{0}^{\infty}(\tilde{\Omega})$, op $_{\varepsilon}(\chi) f^{\varepsilon}$ is uniformly bounded in $L^{2}\left(\mathbf{R}^{3}\right)$ and where $Q$ is given in (16). For $\ell \in\{0,+1,-1\}$, we denote by $\tilde{\Pi}^{\ell}$ the eigenprojectors associated with the eigenvalues

$$
\tilde{\lambda}^{\ell}(s, z, \sigma, \zeta)=\sigma-\ell \sqrt{s^{2}+(\alpha(\zeta, \sigma))^{2} z_{1}^{2}}
$$

of $Q$. The choice of the labelling is made so that $J_{\text {in }}^{ \pm} \cup J_{\text {out }}^{\mp} \subset\left\{\tilde{\lambda}^{ \pm}=0\right\}$. On $I$, one has $z_{1}=0$, therefore if $\left(e_{1}, e_{2}, e_{3}\right)$ is the canonical basis of $\mathbf{C}^{3}$,

$$
\tilde{\Pi}^{0}=e_{3} \otimes e_{3}, \quad \tilde{\Pi}^{-}=\left\{\begin{array}{l}
e_{2} \otimes e_{2} \text { if } s>0, \\
e_{1} \otimes e_{1} \text { if } s<0,
\end{array} \quad \text { and } \quad \tilde{\Pi}^{+}=\left\{\begin{array}{l}
e_{1} \otimes e_{1} \text { if } s>0 \\
e_{2} \otimes e_{2} \text { if } s<0
\end{array}\right.\right.
$$


Let us now investigate the implications of the change of coordinates on the two-scale Wigner measures.

Let $\nu$ be the two-scale Wigner measure associated with the concentration at the scale $\sqrt{\varepsilon}$ of $\left(\psi^{\varepsilon}\right)$ on $I=\{q \wedge p=0\}$ with the choice of coordinates on $N(I)$ of Theorem 1. We recall that these coordinates $(q, t, p, \tau, \eta)$ are such that for $\rho=(q, t, p, \tau) \in I$, the class on $N_{\rho}(I)$ of the vector $\delta \rho=(\delta q, \delta t, \delta p, \delta \tau)$ is characterized by the function

$$
\eta=\delta q \wedge p
$$

Let us denote by $N(\kappa)$ the change of coordinates induced on $N(I)$ by $\kappa$. By the geometric properties of two-scale Wigner measures and Egorov Theorem (see [13]), the measure $\tilde{\nu}=\nu \circ N(\kappa)$ is the two scale Wigner measure of $\left(v^{\varepsilon}\right)$ for $I$ affected with the equation

$$
g(s, z, \sigma, \zeta) z_{1}=0 \text { where } g \circ \kappa^{-1}(q, t, p, \tau)=\frac{|p|}{\lambda(p, \tau) \mu(p, \tau)} .
$$

Besides, $\tilde{\nu}$ decomposes as

$$
\tilde{\nu}=\tilde{\nu}^{0} \tilde{\Pi}^{0}+\tilde{\nu}^{+} \tilde{\Pi}^{+}+\tilde{\nu}^{-} \tilde{\Pi}^{-}
$$

and we are concerned with the link of the traces $\tilde{\nu}_{\text {out }}^{\ell}$ of $\tilde{\nu}^{\ell}$ on $S$ for $s>0$ with the traces $\tilde{\nu}_{i n}^{\ell}$ of $\tilde{\nu}^{\ell}$ on $S$ for $s<0$.

Finally, let us point out that it is not clear that the system (8) is well posed in $s$ (indeed, the function $\alpha$ depends on the variable $\sigma$ ). We address this point in the first subsection, then we successively analyze the two scale Wigner measure above $|\eta|<\infty$ and $|\eta|=\infty$ in the two following subsections.

\subsection{An hyperbolic estimate.}

Proposition 9. Let $\left(v^{\varepsilon}\right)$ be a bounded family of $L^{2}\left(\mathbf{R}_{s} \times \mathbf{R}_{z}^{2}, \mathbf{C}^{3}\right)$ satisfying (8), then if $\phi \in \mathcal{C}_{0}^{\infty}(\mathbf{R})$ with $\phi=1$ close to 0 , there exists $\delta_{0}>0$ and $\varepsilon_{0}>0$ such that the family $\left(\phi\left(\frac{z_{1}}{\delta_{0}}\right) v^{\varepsilon}\right)_{\varepsilon<\varepsilon_{0}}$ is bounded in $L^{\infty}\left(\mathbf{R}_{s}, L^{2}\left(\mathbf{R}_{z}^{2}\right)\right)$.

This proposition is proved in the same manner as Proposition 7 in [13] and implies as in [13] that one can evaluate the action of pseudodifferential operators on functions $\left(v^{\varepsilon}\right)$ as follows.

Lemma 10. There exists a neighborhood $\Omega_{1} \subset \tilde{\Omega}$ of 0 such that for all $\chi \in$ $\mathcal{C}_{0}^{\infty}\left(\Omega_{1}\right)$, the family $\underline{v}^{\varepsilon}=\mathrm{op}_{\varepsilon}(\chi) v^{\varepsilon}$ satisfies:

for $\varepsilon$ small enough and for all compact $\mathcal{K} \subset \mathbf{R}^{6}$, there exists $N \in \mathbf{N}$ such that for all $a \in \mathcal{C}_{0}^{\infty}\left(\mathcal{K}, \mathbf{C}^{3,3}\right)$

$$
\left|\left(\operatorname{op}_{\varepsilon}(a) \underline{v}^{\varepsilon} \mid \underline{v}^{\varepsilon}\right)\right| \leq C \int_{-\infty}^{+\infty} \sup _{k+|\beta| \leq N(z, \sigma, \zeta) \in \mathbf{R}^{5}}\left|\partial_{\sigma}^{k} \partial_{\zeta}^{\beta} a(s, z, \sigma, \zeta)\right| d s .
$$

We refer to [13] for the proofs. For the proof of the Proposition, one just need to replace the matrix $L$ of [13] by the new definition (15) and for the proof of the Lemma, one has to exchange the role of $z$ and $\zeta$. 
REMARK 11. Note that if the symbol of Lemma 10 is of the form a $(s, z, \sigma, \zeta)=$ $a_{0}(z, \sigma, \zeta) \phi\left(\frac{s}{r}\right)$ with $r>0, \phi \in \mathcal{C}_{0}^{\infty}(\mathbf{R})$ and $a_{0} \in \mathcal{C}_{0}^{\infty}\left(\mathbf{R}^{5}\right)$, then

$$
\left|\left(\mathrm{op}_{\varepsilon}(a) \underline{v}^{\varepsilon} \mid \underline{v}^{\varepsilon}\right)\right| \leq C \int_{-\infty}^{+\infty}\left|\phi\left(\frac{s}{r}\right)\right| d s \underset{r \rightarrow 0}{\longrightarrow} 0 .
$$

Therefore, if $\tilde{\mu}$ is a Wigner measure of $\left(v^{\varepsilon}\right)$, we have $\tilde{\mu}(\{s=0\})=0$.

5.2. Analysis at finite distance. We aim at proving Theorem 1 in $\{|\eta|<$ $+\infty\}$, i.e. in $\{|\eta|<R\}$ for any $R>0$. Let us fix $R>0$, we use the two-microlocal normal form of Section 3.2: we localize $\eta$ in the ball $B=\{|\eta| \leq R\}$ of $\mathbf{R}$ applying a cutoff function, $\phi\left(\frac{\eta}{R}\right)$ for $R>0$ and $\phi \in \mathcal{C}_{0}^{\infty}(\mathbf{R})$. Then, applying Proposition 5 (of which we keep the nytations) and setting $V^{\varepsilon}=\mathrm{op}_{\varepsilon}{ }^{I}\left((\operatorname{Id}+\sqrt{\varepsilon} \tilde{C}) \phi\left(\frac{\eta}{R}\right)\right) v^{\varepsilon}$, we obtain

$$
\forall a \in \mathcal{C}_{0}^{\infty}\left(\mathbf{R}^{6} \times B\right), \text { op }_{\varepsilon}{ }^{I}(a) \operatorname{op}_{\varepsilon}\left(Q_{0}\right) V^{\varepsilon}=\varepsilon F^{\varepsilon}(s, z)
$$

where $Q_{0}$ is defined in equation (17) and $\left(F^{\varepsilon}\right)$ is uniformly bounded in $L^{2}\left(\mathbf{R}_{s, z}^{3}\right)$. Obviously, the two-scale Wigner measures of $\left(V^{\varepsilon}\right)$ and $\left(v^{\varepsilon}\right)$ are the same in $B$ and we aim to describe the traces on $s=0^{-}$in terms of the traces on $s=0^{+}$of the two-scale semiclassical measures $\tilde{\nu}$ of $\left(V^{\varepsilon}\right)$. The function $\left(F^{\varepsilon}\right)$ does not contribute to this description. Indeed, denote by $S_{\varepsilon}\left(s, s^{\prime}\right)$ the evolution operator associated with the free system $(37)$ and let $\left(U^{\varepsilon}\right)$ be a solution of this system defined more precisely by,

$$
\mathrm{op}_{\varepsilon}\left(Q_{0}\right) U^{\varepsilon}=0, \quad U_{\mid s=0}^{\varepsilon}=V_{\mid s=0}^{\varepsilon},
$$

then we have

$$
V^{\varepsilon}(s)=U^{\varepsilon}(s)+i \int_{0}^{s} S_{\varepsilon}(0, t) F^{\varepsilon}(t) d t .
$$

Since the family $\left(F^{\varepsilon}\right)$ is uniformly bounded in $L^{2}\left(\mathbf{R}_{s, z}^{3}\right)$, we deduce from Hölder inequality,

$$
V^{\varepsilon}(s)=U^{\varepsilon}(s)+O(\sqrt{|s|}) \text { in } L^{2}\left(\mathbf{R}_{z}^{d}\right) .
$$

Therefore, the traces of the two-scale Wigner measures of $\left(U^{\varepsilon}\right)$ and $\left(V^{\varepsilon}\right)$ on $s=0^{ \pm}$ are the same. In the following, we focus on system (37), which allows to calculate the transfer coefficient in the variables $(s, z, \sigma, \zeta)$ and then, in the variables $(q, t, p, \tau)$.

Let us denote as before by $\tilde{\nu}$ the two-scale Wigner measure of the family $\left(v^{\varepsilon}\right)$ (and thus of $\left(U^{\varepsilon}\right)$ on $\left.I \times B\right)$ for the hypersurface $I=\left\{g(s, z, \sigma, \zeta) z_{1}=0\right\}$ where $g$ is defined in (34). Because of (33) and (35), one can identify the measures $\tilde{\nu}^{0}, \tilde{\nu}^{+}$and $\tilde{\nu}^{-}$with the two-scale Wigner measures of the components of $U^{\varepsilon}$. Set $U^{\varepsilon}=\left(U_{1}^{\varepsilon}, U_{2}^{\varepsilon}, U_{3}^{\varepsilon}\right)$, then $\tilde{\nu}^{0}$ is the two-scale Wigner measure of $\left(U_{3}^{\varepsilon}\right), \tilde{\nu}^{+}$of $\left(U_{1}^{\varepsilon}\right)$ if $s>0$ and of $\left(U_{2}^{\varepsilon}\right)$ if $s<0$ and finally, $\tilde{\nu}^{-}$the measure of $\left(U_{2}^{\varepsilon}\right)$ if $s>0$ and of $\left(U_{1}^{\varepsilon}\right)$ if $s<0$.

The crucial observation is that if one performs a symplectic change of variables $(z, \zeta) \mapsto(\tilde{z}, \tilde{\zeta})$ such that $\tilde{z}_{1}=\alpha(0, \zeta) z_{1}$ and if one sets

$$
\tilde{U}^{\varepsilon}(s, \tilde{z})=K U^{\varepsilon}(\sqrt{\varepsilon} s, \sqrt{\varepsilon} \tilde{z})
$$


where $K$ is a (scalar) Fourier integral operator associated with the symplectic change of coordinates, then $\tilde{U}^{\varepsilon}=\left(V_{+}, V_{-}, V_{0}\right)$ satisfies the system (18) with $z=\tilde{z}_{1}$. Then, the measure $\tilde{\nu}^{+}$in $\{s>0\}$ is the two scale Wigner measure of $\left(V_{+}\right)$. By the scattering Theorem 6 and the relation between $\omega_{+}$in one side and $\alpha_{0}, \alpha_{+}$and $\alpha_{-}$on the other side, we obtain

$$
\nu_{\text {out }}^{+}=\left(1-\mathrm{e}^{-\pi \tilde{\eta}^{2} / 2}\right)^{2} \nu_{i n}^{+}+\mathrm{e}^{-\pi \tilde{\eta}^{2}} \nu_{i n}^{-}+2\left(1-\mathrm{e}^{-\pi \tilde{\eta}^{2} / 2}\right) \mathrm{e}^{-\pi \tilde{\eta}^{2} / 2} \nu_{i n}^{0}
$$

provided that $\nu_{i n}^{0}, \nu_{i n}^{+}$and $\nu_{i n}^{-}$are mutually singular and where the variable $\tilde{\eta}$ characterize the class of the vector $\delta \rho$ in $N_{\rho}(I)$ according to

$$
\tilde{\eta}=\delta \tilde{z}_{1}
$$

Therefore,

$$
\tilde{\eta}=\alpha(0, \zeta) \frac{\eta}{g(s, z, \sigma, \zeta)}
$$

In view of $(34),|\lambda|=|p|^{-1 / 2}, \alpha(\sigma, \zeta)=\mu(\sigma, \zeta)^{-1}$ and of the fact that $\sigma=0$ on $I$, we obtain

$$
\tilde{\eta}=\eta|p|^{-\frac{3}{2}}
$$

Studying similarly $\nu_{\text {out }}^{-}$and $\nu_{\text {out }}^{0}$, we obtain Theorem 1 in $|\eta|<+\infty$.

5.3. Analysis at infinity. It remains to prove Theorem 1 in $\{|\eta|=\infty\}$, i.e. that

$$
\tilde{\nu}_{\text {out }}^{\ell} \mathbf{1}_{|\eta|=\infty}=\tilde{\nu}_{\text {in }}^{\ell} \mathbf{1}_{|\eta|=\infty} \quad \forall \ell \in\{0,+1,-1\} .
$$

Let us argue for the + mode. We follow the strategy of [13] in Section 7.2 and detail the arguments because the fact that we have three modes implies a few complications. Remark 11 shows that $\tilde{\nu}^{+}(\{s=0\})=0$. We consider $r>0, a_{0}=a_{0}(z, \sigma, \zeta) \in$ $\mathcal{C}_{0}^{\infty}\left(\mathbf{R}^{5}\right), \phi \in \mathcal{C}_{0}^{\infty}(\mathbf{R}), \phi=1$ in a neighborhood of $0, \delta<\delta_{0}$ (where $\delta_{0}$ is given by Proposition 9$), R>0$. We suppose $r$ small enough and $\operatorname{supp}\left(a_{0}\right)$ conveniently chosen so that $a(\cdot, \eta)$ is compactly supported in $\Omega_{1}$ for all $\eta \in \mathbf{R}$, where $\tilde{\Omega}_{1}$ is the open set of Lemma 10. Then, we have

$$
\left\langle a_{0}, \tilde{\nu}_{\text {out }}^{+} \mathbf{1}_{|\eta|=\infty}-\tilde{\nu}_{\text {in }}^{-} \mathbf{1}_{|\eta|=\infty}\right\rangle=\lim _{r \rightarrow 0}\left\langle a_{0} \phi\left(\frac{s}{r}\right), \partial_{s} \tilde{\nu}^{+} \mathbf{1}_{|\eta|=+\infty}\right\rangle .
$$

We set

$$
a(s, \sigma, z, \zeta, \eta)=a_{0}(z, \sigma, \zeta) \phi\left(\frac{s}{r}\right) \phi\left(\frac{z_{1}}{\delta}\right)(1-\phi)\left(\frac{\eta}{R}\right)
$$

The function $a$ is a symbol of $\mathcal{A}$ and on the support of $a\left(s, \sigma, z, \zeta, z_{1} / \sqrt{\varepsilon}\right)$, one has $\left|z_{1}\right|>R \sqrt{\varepsilon}$ so that the function $a \tilde{\Pi}^{+}$is smooth, thus the operator op ${ }_{\varepsilon}^{I}\left(a \tilde{\Pi}^{+}\right)$is well defined. Besides,

$$
\left\langle a_{0} \phi\left(\frac{s}{r}\right), \partial_{s} \tilde{\nu}^{+} \mathbf{1}_{|\eta|=+\infty}\right\rangle=\lim _{R \rightarrow+\infty} \lim _{\delta \rightarrow 0} \lim _{\varepsilon \rightarrow 0}\left(\operatorname{op}_{\varepsilon}^{I}\left(\partial_{s} a \tilde{\Pi}^{+}\right) v^{\varepsilon}, v^{\varepsilon}\right) .
$$

Therefore, we set

$$
L_{\varepsilon}=\left(\operatorname{op}_{\varepsilon}^{I}\left(\partial_{s} a \tilde{\Pi}^{+}\right) v^{\varepsilon}, v^{\varepsilon}\right)
$$


and we shall study $L_{\varepsilon}$ as first $\varepsilon \rightarrow 0$, then $\delta \rightarrow 0, R \rightarrow+\infty$ and finally $r \rightarrow 0$.

We remark that

$$
\mathrm{op}_{\varepsilon}{ }^{I}\left(\partial_{s} a \tilde{\Pi}^{+}\right)=\frac{i}{\varepsilon}\left[\frac{\varepsilon}{i} \partial_{s}, \mathrm{op}_{\varepsilon}{ }^{I}\left(a \tilde{\Pi}^{+}\right)\right]-\mathrm{op}_{\varepsilon}{ }^{I}\left(a \partial_{s} \tilde{\Pi}^{+}\right) .
$$

Therefore, using the equation, we can write $L_{\varepsilon}=L_{\varepsilon}^{1}+L_{\varepsilon}^{2}+L_{\varepsilon}^{3}$ with

$$
\begin{aligned}
L_{\varepsilon}^{1} & =-i\left(\mathrm{op}_{\varepsilon}{ }^{I}\left(a \tilde{\Pi}^{+}\right) v^{\varepsilon}, f^{\varepsilon}\right)+i\left(\mathrm{op}_{\varepsilon}{ }^{I}\left(a \tilde{\Pi}^{+}\right) f^{\varepsilon}, v^{\varepsilon}\right) \\
L_{\varepsilon}^{2} & =\frac{i}{\varepsilon}\left(\left[\operatorname{op}_{\varepsilon}\left(V_{P J T}\left(s, \alpha(\sigma, \zeta) z_{1}\right)\right), \mathrm{op}_{\varepsilon}{ }^{I}\left(a \tilde{\Pi}^{+}\right)\right] v^{\varepsilon}, v^{\varepsilon}\right) \\
L_{\varepsilon}^{3} & =\left(\operatorname{op}_{\varepsilon}{ }^{I}\left(a \partial_{s} \tilde{\Pi}^{+}\right) v^{\varepsilon}, v^{\varepsilon}\right) .
\end{aligned}
$$

We now prove successively that these three terms go to 0 in the prescribed limit. We will use symbolic calculus and for that, we introduce some notations. If $q=$ $q(s, z, \sigma, \zeta, \eta)$, we set

$$
q_{\varepsilon}(s, z, \sigma, \zeta)=q\left(s, z, \sigma, \zeta, \frac{z_{1}}{\sqrt{\varepsilon}}\right) \text { and } q_{\varepsilon}^{\sharp}(s, z, \sigma, \zeta)=q\left(s, z, \varepsilon \sigma, \varepsilon \zeta, \frac{z_{1}}{\sqrt{\varepsilon}}\right)
$$

so that we have

$$
\mathrm{op}_{\varepsilon}{ }^{I}(q)=\mathrm{op}_{\varepsilon}\left(q_{\varepsilon}\right)=\mathrm{op}_{1}\left(q_{\varepsilon}^{\sharp}\right) .
$$

We observe that $\tilde{\Pi}^{+}$is homogeneous in the variables $\left(s, \alpha(\sigma, \zeta) z_{1}\right)$, thus for $q=a \tilde{\Pi}^{+}$, we have

$$
\left|\partial_{\sigma, z_{2}, \zeta}^{\beta} \partial_{s, z_{1}}^{\gamma} q_{\varepsilon}(s, z, \sigma, \zeta)\right| \leq C_{\beta, \gamma}\left(\frac{1}{R \sqrt{\varepsilon}}\right)^{|\gamma|}
$$

where we have used that

$$
\theta\left(s, z_{1}, \sigma, \zeta\right):=\sqrt{s^{2}+\alpha(\sigma, \zeta)^{2} z_{1}^{2}} \geq R \sqrt{\varepsilon}
$$

on the support of $q_{\varepsilon}$ and where we implicitely assume $R \sqrt{\varepsilon} \leq 1$. In terms of WeylHörmander metric (ses sections 18.4, 18.5, 18.6 of [21]), one has $q_{\varepsilon}^{\sharp} \in S\left(1, g_{\varepsilon}\right.$ ), where $g_{\varepsilon}$ is the metric

$$
g_{\varepsilon}=d z_{2}^{2}+\frac{d s^{2}+d z_{1}^{2}}{R^{2} \varepsilon}+\varepsilon^{2}\left(d \sigma^{2}+d \zeta^{2}\right)
$$

Then

$$
\frac{g_{\varepsilon}}{g_{\varepsilon}^{\sigma}} \leq\left(\frac{\sqrt{\varepsilon}}{R}\right)^{2}
$$

so that the gain of the symbolic calculus is of order $\frac{\sqrt{\varepsilon}}{R}$.

- Analysis of $L_{\varepsilon}^{1}$ : Since the function $f^{\varepsilon}$ is locally bounded in $L^{2}$, we consider $\chi \in \mathcal{C}_{0}^{\infty}\left(\Omega_{1}\right)$ such that $\chi=1$ on the support of $a$ (as before the open set $\Omega_{1}$ is the one of Lemma 10). Then the symbolic calculus Theorem 18.5.4 of [21] gives

$$
\mathrm{op}_{\varepsilon}{ }^{I}\left(a \tilde{\Pi}^{+}\right)=\mathrm{op}_{\varepsilon}{ }^{I}\left(a \tilde{\Pi}^{+} \chi\right) \in \mathrm{op}_{\varepsilon}^{I}\left(a \tilde{\Pi}^{+}\right) \mathrm{op}_{\varepsilon}(\chi)+\mathrm{op}_{1}\left(S\left(\left(\frac{\sqrt{\varepsilon}}{R}\right)^{N}, g_{\varepsilon}\right)\right)
$$


for all $N \in \mathbf{N}$. Then, the $L^{2}$ continuity Theorem 18.6.3 of [21] implies as $\varepsilon \rightarrow 0$,

$$
\left(\operatorname{op}_{\varepsilon}{ }^{I}\left(a \tilde{\Pi}^{+}\right) f^{\varepsilon} \mid v^{\varepsilon}\right)=\left(\operatorname{op}_{\varepsilon}{ }^{I}\left(a \tilde{\Pi}^{+}\right) \operatorname{op}_{\varepsilon}(\chi) f^{\varepsilon} \mid v^{\varepsilon}\right)+o(1) .
$$

Lemma 10 and the boundedness in $L^{2}$ of op op $(\chi) f^{\varepsilon}$ give the existence of $C>0$ such that

$$
\left|\left(\operatorname{op}_{\varepsilon}{ }^{I}\left(a \tilde{\Pi}^{+}\right) \operatorname{op}_{\varepsilon}(\chi) f^{\varepsilon} \mid v^{\varepsilon}\right)\right| \leq C \int_{-\infty}^{+\infty}\left|\phi\left(\frac{s}{r}\right)\right| \mathrm{d} s \underset{r \rightarrow 0}{\longrightarrow} 0 .
$$

One argues similarly with the other term.

- Analysis of $L_{\varepsilon}^{2}$ : We first observe that $\left(V_{P J T}\left(s, \alpha(\sigma, \zeta) z_{1}\right)\right)_{\varepsilon}^{\sharp} \in S\left(\theta_{\varepsilon}^{\sharp}, g_{\varepsilon}\right)$ where $\theta$ is defined in (38) and that $q_{\varepsilon}^{\sharp} \in S\left(\left(\theta_{\varepsilon}^{\sharp}\right)^{-N}, g_{\varepsilon}\right)$ for all $N \in \mathbf{N}$ because $a$ is supported in a fixed compact set. Therefore, using that $V_{P J T}\left(s, \alpha(\sigma, \zeta) z_{1}\right)$ and $\tilde{\Pi}^{+}$commute, we obtain by symbolic calculus

$$
\begin{array}{r}
\frac{1}{\varepsilon}\left[\operatorname{op}_{1}\left(\left(V_{P J T}\left(s, \alpha(\sigma, \zeta) z_{1}\right)\right)_{\varepsilon}^{\sharp}\right), \mathrm{op}_{1}\left(q_{\varepsilon}^{\sharp}\right)\right] \in \mathrm{op}_{1}\left(\frac{1}{2 i \varepsilon}\left\{\left(V_{P J T}\left(s, \alpha(\sigma, \zeta) z_{1}\right)\right)_{\varepsilon}^{\sharp},\left(a \tilde{\Pi}^{+}\right)_{\varepsilon}^{\sharp}\right\}\right. \\
\left.-\frac{1}{2 i \varepsilon}\left\{\left(a \tilde{\Pi}^{+}\right)_{\varepsilon}^{\sharp},\left(V_{P J T}\left(s, \alpha(\sigma, \zeta) z_{1}\right)\right)_{\varepsilon}^{\sharp}\right\}\right)+\frac{1}{\varepsilon} \mathrm{op}_{1}\left(S\left(\left(\frac{\sqrt{\varepsilon}}{R}\right)^{2}, g_{\varepsilon}\right)\right) .
\end{array}
$$

It is at this very place that the proof differs from the one of [13] because of the presence of the three modes which induces the presence of additional terms. We observe that

$$
\begin{aligned}
& \frac{1}{\varepsilon}\left\{\left(V_{P J T}\left(s, \alpha(\sigma, \zeta) z_{1}\right)\right)_{\varepsilon}^{\sharp},\left(a \tilde{\Pi}^{+}\right)_{\varepsilon}^{\sharp}\right\} \\
= & \left(\left\{V_{P J T}\left(s, \alpha(\sigma, \zeta) z_{1}\right),\left(a \tilde{\Pi}^{+}\right)\right\}_{s, z, \sigma, \zeta}\right)_{\varepsilon}^{\sharp}+\frac{1}{R \sqrt{\varepsilon}}\left(\partial_{\eta} a V_{P J T}\left(0, \partial_{\zeta_{1}} \alpha(\sigma,, \zeta) z_{1}\right) \tilde{\Pi}^{+}\right)_{\varepsilon}^{\sharp} \\
= & \left(\left\{V_{P J T}\left(s, \alpha(\sigma, \zeta) z_{1}\right),\left(a \tilde{\Pi}^{+}\right)\right\}_{s, z, \sigma, \zeta}\right)_{\varepsilon}^{\sharp}+\frac{1}{R}\left(\partial_{\eta} a V_{P J T}\left(0, \partial_{\zeta_{1}} \alpha(\sigma, \zeta) \eta\right) \tilde{\Pi}^{+}\right)_{\varepsilon}^{\sharp} .
\end{aligned}
$$

Similarly,

$$
\begin{aligned}
& \frac{1}{\varepsilon}\left\{\left(a \tilde{\Pi}^{+}\right)_{\varepsilon}^{\sharp},\left(V_{P J T}\left(s, \alpha(\sigma, \zeta) z_{1}\right)\right)_{\varepsilon}^{\sharp}\right\} \\
= & \left(\left\{\left(a \tilde{\Pi}^{+}\right), V_{P J T}\left(s, \alpha(\sigma, \zeta) z_{1}\right)\right\}_{s, z, \sigma, \zeta}\right)_{\varepsilon}^{\sharp}+\frac{1}{R}\left(\partial_{\eta} a \tilde{\Pi}^{+} V_{P J T}\left(0, \partial_{\zeta_{1}} \alpha(\sigma, \zeta) \eta\right)\right)_{\varepsilon}^{\sharp} .
\end{aligned}
$$

Therefore $L_{\varepsilon}^{2}=\left(\operatorname{op}_{\varepsilon}{ }^{I}(B) v^{\varepsilon}, v^{\varepsilon}\right)+O\left(R^{-2}\right)$ with

$$
\begin{aligned}
B= & \left\{V_{P J T}\left(s, \alpha(\sigma, \zeta) z_{1}\right), a \tilde{\Pi}^{+}\right\}_{s, z, \sigma, \zeta}-\left\{a \tilde{\Pi}^{+}, V_{P J T}\left(s, \alpha(\sigma, \zeta) z_{1}\right)\right\}_{s, z, \sigma, \zeta} \\
& +\frac{1}{R} \partial_{\eta} a\left(\tilde{\Pi}^{+} V_{P J T}\left(0, \partial_{\zeta_{1}} \alpha(\sigma, \zeta) \eta\right)+V_{P J T}\left(0, \partial_{\zeta_{1}} \alpha(\sigma, \zeta) \eta\right) \tilde{\Pi}^{+}\right) .
\end{aligned}
$$

We write $B=B_{1}+B_{2}+B_{3}$ with

$$
B_{1}=\left\{V_{P J T}, a\right\} \tilde{\Pi}^{+}-\tilde{\Pi}^{+}\left\{a, V_{P J T}\right\} \text { and } B_{2}=a\left(\left\{V_{P J T}, \tilde{\Pi}^{+}\right\}-\left\{\tilde{\Pi}^{+}, V_{P J T}\right\}\right) .
$$

The matrix $B_{1}+B_{3}$ is such that by Lemma 10, we have

$$
\left|\left(\operatorname{op}_{\varepsilon}^{I}\left(B_{1}+B_{3}\right) v^{\varepsilon}, v^{\varepsilon}\right)\right| \leq C \int_{-\infty}^{+\infty}\left(\frac{\delta}{r}\left|\phi^{\prime}\left(\frac{s}{r}\right)\right|+\phi\left(\frac{s}{r}\right)\right) d s
$$


The term in $B_{2}$ is more complicated and we cut it into two parts. Indeed, using

$$
V_{P J T}\left(s, \alpha(\sigma, \zeta) z_{1}\right)=\theta\left(\tilde{\Pi}^{+}-\tilde{\Pi}^{-}\right)
$$

we write

$$
\begin{aligned}
B_{2}= & a \theta\left(\left\{\tilde{\Pi}^{+}-\tilde{\Pi}^{-}, \tilde{\Pi}^{+}\right\}-\left\{\tilde{\Pi}^{+}, \tilde{\Pi}^{+}-\tilde{\Pi}^{-}\right\}\right) \\
& +a\left(\left(\tilde{\Pi}^{+}-\tilde{\Pi}^{-}\right)\left\{\theta, \tilde{\Pi}^{+}\right\}-\left\{\tilde{\Pi}^{+}, \theta\right\}\left(\tilde{\Pi}^{+}-\tilde{\Pi}^{-}\right)\right) \\
= & a \theta\left(\left\{\tilde{\Pi}^{+}, \tilde{\Pi}^{-}\right\}-\left\{\tilde{\Pi}^{-}, \tilde{\Pi}^{+}\right\}\right)+a\left(\left(\tilde{\Pi}^{+}-\tilde{\Pi}^{-}\right)\left\{\theta, \tilde{\Pi}^{+}\right\}+\left\{\theta, \tilde{\Pi}^{+}\right\}\left(\tilde{\Pi}^{+}-\tilde{\Pi}^{-}\right)\right) .
\end{aligned}
$$

We shall prove that $B_{2}$ is off-diagonal where we say that the matrix $D$ is off-diagonal if $\Pi^{\ell} D \Pi^{\ell}=0$ for any $\ell \in\{0,+1,-1\}$. We will study those terms together with $L_{\varepsilon}^{3}$ since $\partial_{s} \tilde{\Pi}^{+}$is also off-diagonal.

Set $B_{2}=B_{2,1}+B_{2,2}$ with

$$
B_{2,2}=a \theta\left(\left\{\tilde{\Pi}^{+}, \tilde{\Pi}^{-}\right\}-\left\{\tilde{\Pi}^{-}, \tilde{\Pi}^{+}\right\}\right) .
$$

We remark that the fact that $\tilde{\Pi}^{\ell} \tilde{\Pi}^{\ell^{\prime}}=\delta_{\ell, \ell^{\prime}} \tilde{\Pi}^{\ell}$ for all $\ell, \ell^{\prime} \in\{0,+1,-1\}$ yields

$$
\forall \ell \in\{0,+1,-1\}, \quad \forall h \in \mathcal{C}^{\infty}\left(\mathbf{R}^{6}\right), \Pi^{\ell}\left\{h, \Pi^{ \pm}\right\} \Pi^{\ell}=0 .
$$

Therefore, applying (39) to $h=\theta$, the matrix $B_{2,1}$ is of the form $B_{2}=a D$ with $D$ off-diagonal.

Let us now consider $B_{2,2}$. Using the general fact $A\{B, C\}-\{A, B\} C=\{A B, C\}-$ $\{A, B C\}$, we observe that for $\ell \neq k, \ell, k \in\{0,+1,-1\}$

$$
\begin{aligned}
\left\{\tilde{\Pi}^{\ell}, \tilde{\Pi}^{k}\right\} & =\left\{\left(\tilde{\Pi}^{\ell}\right)^{2}, \tilde{\Pi}^{k}\right\}=\tilde{\Pi}^{\ell}\left\{\tilde{\Pi}^{\ell}, \tilde{\Pi}^{k}\right\}-\left\{\tilde{\Pi}^{\ell}, \tilde{\Pi}^{\ell}\right\} \tilde{\Pi}^{k}, \\
-\left\{\tilde{\Pi}^{k}, \tilde{\Pi}^{\ell}\right\} & =-\left\{\tilde{\Pi}^{k},\left(\tilde{\Pi}^{\ell}\right)^{2}\right\}=\tilde{\Pi}^{k}\left\{\tilde{\Pi}^{\ell}, \tilde{\Pi}^{\ell}\right\}-\left\{\tilde{\Pi}^{k}, \tilde{\Pi}^{\ell}\right\} \tilde{\Pi}^{\ell},
\end{aligned}
$$

yielding $\tilde{\Pi}^{0}\left\{\tilde{\Pi}^{+}, \tilde{\Pi}^{-}\right\} \tilde{\Pi}^{0}=-\tilde{\Pi}^{0}\left\{\tilde{\Pi}^{-}, \tilde{\Pi}^{+}\right\} \tilde{\Pi}^{0}=0$ and

$$
\begin{aligned}
& \tilde{\Pi}^{-}\left\{\tilde{\Pi}^{+}, \tilde{\Pi}^{-}\right\} \tilde{\Pi}^{-}=-\tilde{\Pi}^{-}\left\{\tilde{\Pi}^{+}, \tilde{\Pi}^{+}\right\} \tilde{\Pi}^{-} \text {and } \tilde{\Pi}^{+}\left\{\tilde{\Pi}^{+}, \tilde{\Pi}^{-}\right\} \tilde{\Pi}^{+}=-\tilde{\Pi}^{+}\left\{\tilde{\Pi}^{-}, \tilde{\Pi}^{-}\right\} \tilde{\Pi}^{+}, \\
& \tilde{\Pi}^{-}\left\{\tilde{\Pi}^{-}, \tilde{\Pi}^{+}\right\} \tilde{\Pi}^{-}=-\tilde{\Pi}^{-}\left\{\tilde{\Pi}^{+}, \tilde{\Pi}^{+}\right\} \tilde{\Pi}^{-} \text {and } \tilde{\Pi}^{+}\left\{\tilde{\Pi}^{-}, \tilde{\Pi}^{+}\right\} \tilde{\Pi}^{+}=-\tilde{\Pi}^{+}\left\{\tilde{\Pi}^{-}, \tilde{\Pi}^{-}\right\} \tilde{\Pi}^{+},
\end{aligned}
$$

We obtain $\tilde{\Pi}^{\ell} B_{2,2} \tilde{\Pi}^{\ell}=0$ for all $\ell \in\{0,+1,-1\}$ and $B_{2,2}$ also is off-diagonal.

- Analysis of $L_{\varepsilon}^{3}$ : Equation (39) for $h=\sigma$ implies that $\partial_{s} \tilde{\Pi}^{+}$is off-diagonal. We now consider terms of the form

$$
L_{\varepsilon}^{3}=\left(\mathrm{op}_{\varepsilon}^{I}(a D) v^{\varepsilon} \mid v^{\varepsilon}\right)
$$

for some matrix $D$ homogeneous of degree -1 in the variables $\left(s, \alpha(\sigma, \zeta) z_{1}\right)$ and such that $\tilde{\Pi}^{\ell} D \tilde{\Pi}^{\ell}=0$ for all $\ell \in\{0,+1,-1\}$. Without loss of generality we can suppose that $D=\tilde{\Pi}^{\ell} D \tilde{\Pi}^{\ell^{\prime}}$ for some $\ell, \ell^{\prime} \in\{0,+1,-1\}$ with $\ell \neq \ell^{\prime}$. Then there exists a real number $c_{\ell, \ell^{\prime}}\left(c_{\ell, \ell^{\prime}} \in\{ \pm 1, \pm 2\}\right)$ such that

$$
\left[D, V_{P J T}\right]=c_{\ell, \ell^{\prime}} \theta D \text {. }
$$


We use this relation to write

$$
a D=\left[\frac{a D}{c_{\ell, \ell^{\prime}} \theta}, V_{P J T}\right]=\left[\frac{a D}{c_{\ell, \ell^{\prime}} \theta},-\sigma \mathrm{Id}+V_{P J T}\right],
$$

which allows to reuse the equation. Again by Weyl-Hörmander calculus with the metric $g_{\varepsilon}$, we obtain $\left(V_{P J T}\right)_{\varepsilon}^{\sharp} \in S\left(\theta_{\varepsilon}^{\sharp}, g_{\varepsilon}\right)$ and

$$
\left(\frac{a D}{c_{\ell, \ell^{\prime}} \theta}\right)_{\varepsilon}^{\sharp} \in S\left(\frac{1}{\sqrt{\varepsilon} R \theta_{\varepsilon}^{\sharp}}, g_{\varepsilon}\right)
$$

(again because of $\theta_{\varepsilon}^{\sharp} \geq C \sqrt{\varepsilon} R$ on the support of $a_{\varepsilon}^{\sharp}$ ). Therefore, we have

$$
\begin{array}{r}
\mathrm{op}_{\varepsilon}{ }^{I}(a D) \in \mathrm{op}_{\varepsilon}\left(-\sigma+V_{P J T}\right) \mathrm{op}_{\varepsilon}{ }^{I}\left(\frac{a D}{c_{\ell, \ell^{\prime}} \theta}\right)-\mathrm{op}_{\varepsilon}{ }^{I}\left(\frac{a D}{c_{\ell, \ell^{\prime}} \theta}\right) \mathrm{op}_{\varepsilon}\left(-\sigma+V_{P J T}\right) \\
+\frac{\varepsilon}{i} \mathrm{op}_{\varepsilon}{ }^{I}\left(\partial_{s}\left(\frac{a D}{c_{\ell, \ell^{\prime}} \theta}\right)\right)+\mathrm{op}_{1}\left(S\left(\frac{1}{R^{2}}, g_{\varepsilon}\right)\right)
\end{array}
$$

whence, using the equation for $v^{\varepsilon}$,

$$
\begin{aligned}
L_{\varepsilon}^{3}=O\left(\frac{1}{R^{2}}\right)+\varepsilon\left(\mathrm{op}_{\varepsilon}^{I}\left(\frac{a D}{c_{\ell, \ell^{\prime}} \theta}\right) v^{\varepsilon}\right. & \left.\mid f^{\varepsilon}\right)-\varepsilon\left(\mathrm{op}_{\varepsilon}^{I}\left(\frac{a D}{c_{\ell, \ell^{\prime}} \theta}\right) f^{\varepsilon} \mid v^{\varepsilon}\right) \\
& +\frac{\varepsilon}{i}\left(\operatorname{op}_{\varepsilon}{ }^{I}\left(\partial_{s}\left(\frac{a D}{c_{\ell, \ell^{\prime}} \theta}\right)\right) v^{\varepsilon} \mid v^{\varepsilon}\right) .
\end{aligned}
$$

Arguing as before, one gets as $\varepsilon$ goes to 0 then $R$ to $+\infty$,

$$
L_{\varepsilon}^{3}=o(1)+O\left(\frac{1}{R^{2}}\right)+\frac{\varepsilon}{i}\left(\operatorname{op}_{\varepsilon}{ }^{I}\left(\partial_{s}\left(\frac{a D}{c_{\ell, \ell^{\prime}} \theta}\right)\right) v^{\varepsilon} \mid v^{\varepsilon}\right) .
$$

Observing that

$$
\left|\partial_{\sigma, \zeta}^{\beta} \partial_{s}\left(\frac{a D}{c_{\ell, \ell^{\prime}} \theta}\right)_{\varepsilon}\right| \leq \frac{C_{\beta}}{\left(s^{2}+\varepsilon R^{2}\right)^{3 / 2}}
$$

we obtain by Lemma 10

$$
\left|L_{\varepsilon}^{3}\right| \leq o(1)+O\left(\frac{1}{R^{2}}\right)+C \varepsilon \int_{-\infty}^{+\infty} \frac{d s}{\left(s^{2}+\varepsilon R^{2}\right)^{3 / 2}} \leq o(1)+O\left(\frac{1}{R^{2}}\right),
$$

which finishes the proof.

Appendix A. Complex wedge product and solutions of ODEs. We consider $(\mathcal{H},\langle\cdot \mid \cdot\rangle)$ an Hilbert space of dimension 3 over $\mathbf{C}$ and $A(s)$ a continuous family of selfadjoint endomorphisms on $\mathcal{H}$. We consider the Schrödinger system

$$
-i \partial_{s} V=A(s) V \text {. }
$$

Let $[\cdot, \cdot, \cdot]$ denote a non trivial alternating trilinear form on $\mathcal{H}$ and let $u_{1}$ and $u_{2}$ be two vectors of $\mathcal{H}$. We define the wedge product of $u_{1}$ and $u_{2}$ with respect to $[\cdot, \cdot, \cdot]$ to be the unique vector $u_{1} \wedge u_{2}$ (through Riesz representation theorem) such that

$$
\forall w \in \mathcal{H}, \quad\left[u_{1}, u_{2}, w\right]=\left\langle u_{1} \wedge u_{2} \mid w\right\rangle .
$$

Then, we have the two following points: 
1. If $A$ is an endomorphism of $\mathcal{H}$, we have

$$
\left[A u_{1}, u_{2}, u_{3}\right]+\left[u_{1}, A u_{2}, u_{3}\right]+\left[u_{1}, u_{2}, A u_{3}\right]=(\operatorname{Tr} A)\left[u_{1}, u_{2}, u_{3}\right] .
$$

2. If $u$ (respectively $v$ ) has coordinates $\left(u_{1}, u_{2}, u_{3}\right)$ (respectively $\left.\left(v_{1}, v_{2}, v_{3}\right)\right)$ in some orthonormal basis $\left(e_{1}, e_{2}, e_{3}\right)$ of $\mathbf{C}^{3}$ then the coordinates of $u \wedge v$ are

$$
\overline{\left[e_{1}, e_{2}, e_{3}\right]\left(u_{2} v_{3}-u_{3} v_{2}, u_{3} v_{1}-u_{1} v_{3}, u_{1} v_{2}-u_{2} v_{1}\right)} \text {. }
$$

TheOREM 12. Let $V_{1}(s)$ and $V_{2}(s)$ be two solutions of the system (41). Then

$$
e^{i \int_{s_{0}}^{s} \operatorname{Tr} A\left(s^{\prime}\right) d s^{\prime}} V_{1}(s) \wedge V_{2}(s)
$$

is also a solution of (41). In particular, if $V_{1}$ and $V_{2}$ are linearly independent, we have a basis of solutions.

Note that this is in fact a corollary of Liouville theorem on Wronskian combined with the fact that the equation is norm preserving.

Proof. If $V_{1}$ and $V_{2}$ are linearly dependent, the wedge product is zero and is certainly a solution. Thus, we can assume that $V_{1}$ and $V_{2}$ are linearly independent so that $\left(V_{1}(s), V_{2}(s), V_{1}(s) \wedge V_{2}(s)\right)$ is an instantaneous basis of $\mathcal{H}$. Removing the phase, it is enough to show that $W(s)=V_{1}(s) \wedge V_{2}(s)$ is a solution of

$$
-i \partial_{s} W=[A(s)-\operatorname{Tr} A(s) \operatorname{Id}] W
$$

which we will show to hold in the preceding instantaneous basis. We first have

$\left\langle V_{j}(s) \mid\left(-i \partial_{s} W\right)(s)\right\rangle=\left\langle-i \partial_{s} V_{j} \mid W\right\rangle=\left\langle A V_{j} \mid W\right\rangle=\left\langle V_{j} \mid A W\right\rangle=\left\langle V_{j}(s) \mid[A(s)-\operatorname{Tr} A(s) \operatorname{Id}] W(s)\right\rangle$

where we have used that $V_{j}$ and $W$ are orthogonal, the equation and the selfadjointness of $A(s)$. Similarly, using (42) and (43) we obtain

$$
\begin{aligned}
\left\langle W(s) \mid\left(-i \partial_{s} W\right)(s)\right\rangle & =-\left\langle W \mid\left(-i \partial_{s} V_{1}\right) \wedge V_{2}\right\rangle-\left\langle W \mid V_{1} \wedge\left(-i \partial_{s} V_{2}\right)\right\rangle \\
& =-\left\langle W \mid A V_{1} \wedge V_{2}\right\rangle-\left\langle W \mid V_{1} \wedge A V_{2}\right\rangle \\
& =-\overline{\left[A V_{1}, V_{2}, W\right]}-\overline{\left[V_{1}, A V_{2}, W\right]} \\
& =\overline{\left[V_{1}, V_{2}, A W\right]-(\operatorname{Tr} A)\left[V_{1}, V_{2}, W\right]} \\
& =\left\langle[A-\operatorname{Tr} A \operatorname{Id}] W \mid V_{1} \wedge V_{2}\right\rangle \\
& =\langle W(s) \mid[A(s)-\operatorname{Tr} A(s) \operatorname{Id}] W(s)\rangle .
\end{aligned}
$$

Acknowledgements. The authors thank A. Joye, P. Gérard and C. Lasser for friendly and fruitful discussions.

\section{REFERENCES}

[1] M. Born And R. Oppenheimer, Zur Quantentheorie der Molekeln, Ann. Phys. (Leipzig), 84 (1927), pp. 457-484.

[2] S. BRundobler AND V. Elser, S-matrix for generalized Landau-Zener problem, J. Phys. A, 26 (1993), pp. 1211-1227. 
[3] C.E. Caroll and F.T. Hioe, Generalisation of the Landau-Zener calculation to three levels, J. Phys. A, 19 (1986), pp. 1151-1161.

[4] Y. COLIN DE VerdiÈRe, The level crossing problem in semi-classical analysis. I. The symmetric case, Proceedings of the International Conference in Honor of Frédéric Pham (Nice, 2002), Ann. Inst. Fourier (Grenoble), 53:4 (2003), pp. 1023-1054.

[5] Y. Colin de Verdière, The level crossing problem in semi-classical analysis. II. The Hermitian case, Ann. Inst. Fourier (Grenoble), 54:5 (2004), pp. 1423-1441.

[6] J. Dieudonné, Calcul infinitésimal, Hermann Paris, 1980.

[7] M. Dimassi AND J. SJöstrand, Spectral asymptotics in the semi-classical limit, London Mathematical Society Lecture Note Series, 268, Cambridge University Press, 1999.

[8] W. Domcke, D. Yarkony and H. Köppel, Conical intersections, World Scientific Publishing, 2004.

[9] T. Duyckaerts, C. Fermanian Kammerer and T. Jecko, Degenerated codimension 1 crossings and resolvent estimates, Asymptot. Anal., 65:3-4 (2009), pp. 147-174.

[10] W. Eisfeld And A. Viel, Higher order $(A+E) \otimes$ e pseudo Jahn-Teller coupling, J. Chem. Phys., 122 (2005), 204317.

[11] C. Fermanian KAmmerer, Wigner measures and molecular propagation through generic energy level crossings, Reviews in Mathematical Physics, 15 (2003), pp. 1285-1317.

[12] C. Fermanian Kammerer, Normal forms for conical intersections in quantum chemistry, Math. Phys. Elect. Jour., 13:4 (2007).

[13] C. Fermanian Kammerer And P. Gérard, Mesures semi-classiques et croisements de modes, Bull. Soc. math. France, 130:1 (2002), pp. 145-190.

[14] C. Fermanian Kammerer and P. Gérard, A Landau-Zener formula for non-degenerated involutive codimension 3 crossings, Ann. Henri Poincaré, 4 (2003), pp. 513-552.

[15] C. Fermanian Kammerer and C. Lasser, Propagation through generic level crossings: a surface hopping semigroup, SIAM J. of Math. Anal., 140:1 (2008), pp. 103-133.

[16] C. Fermanian Kammerer And C. Lasser, Single switch surface hopping for molecular dynamics with transitions, Journal of Chemical Physics, 128 (2008), 144102.

[17] P. GÉRARd And E. Leichtnam, Ergodic properties of eigenfunctions for the Dirichlet problem, Duke Math. J., 71:2 (1993), pp. 559-607.

[18] P. Gérard, P. Markowich, N. Mauser and F. Poupaud, Homogenization limits and Wigner transforms, Commun. Pure Appl. Math., 50:4 (1997), pp. 323-379; and 53 (2000), pp. 280281.

[19] G.A. Hagedorn, Molecular Propagation through Electron Energy Level Crossings, Mem. Am. Math. Soc., 111, no. 536, 1994.

[20] G.A. Hagedorn And A. Joye, Landau-Zener transitions through small electronic eigenvalue gaps in the Born-Oppenheimer approximation, Ann. Inst. Henri Poincaré, 68:1 (1998), pp. $85-134$.

[21] L. Hörmander, The analysis of linear partial differential operators III. Pseudo-differential operators, Classics in Mathematics, Springer, 1985.

[22] L. Landau, Collected papers of L. Landau, Pergamon Press, 1965.

[23] C. Lasser, T. Swart, And S. Teufel, Construction and validation of a rigorous surface hopping algorithm for conical crossings, Commun. Math. Sci., 5 (2007), pp. 789-814.

[24] C. Lasser and S. Teufel, Propagation through Conical Crossings: an Asymptotic Semigroup, Commun. Pure Appl. Math., 58:9 (2005), pp. 1188-1230.

[25] P.-L. Lions And T. PAUl, Sur les mesures de Wigner, Rev. Mat. Iberoamericana, 9:3 (1993), pp. 553-618.

[26] L. Miller, Propagation d'onde semi-classiques à travers une interface et mesures 2microlocales, Thèse de l'Ecole Polytechnique (1996).

[27] D. Robert, Autour de l'approximation semi-classique, Birkhaüser, (1983).

[28] H. Spohn And S. Teufel, Adiabatic decoupling and time-dependent Born-Oppenheimer theory, Commun. Math. Phys., 224:1 (2001), pp. 113-132.

[29] J. Tully And R. Preston, Trajectory surface hopping approach to nonadiabatic molecular collisions: the reaction of $\mathrm{H}^{+}$with $\mathrm{D}_{2}$, J. Chem. Phys., 55:2 (1971), pp. 562-572.

[30] A. Viel, W. Eisfeld, S. Neumann, W. Domcke and U. Manthe, Photoionization-induced dynamics of ammonia: Ab initio potential energy surfaces and time-dependent wave packet calculations for the ammonia cation, J. Chem. Phys., 124 (2006), 214306.

[31] C. Zener, Non-adiabatic crossing of energy levels, Proc. Roy. Soc. Lond., 137 (1932), pp. 696702 . 
